\title{
Analysis and prediction of the impact of technological parameters on cutting force components in rough milling of AZ31 magnesium alloy
}

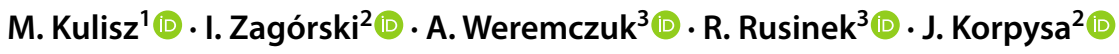

Received: 21 April 2021 / Revised: 18 September 2021 / Accepted: 3 October 2021 / Published online: 21 October 2021

(c) The Author(s) 2021

\begin{abstract}
This paper presents the results of experimental study of the AZ31 magnesium alloy milling process. Dry milling was carried out under high-speed machining conditions. First, a stability lobe diagram was determined using CutPro software. Next, experimental studies were carried out to verify the stability lobe diagram. The tests were carried out for different feed per tooth and cutting speed values using two types of tools. During the experimental investigations, cutting forces in three directions were recorded. The obtained time series were subjected to general analysis and analysis using composite multiscale entropy. Modelling and prediction were performed using Statistica Neural Network software, in which two types of neural networks were applied: multi-layered perceptron and radial basis function. It was observed that milling with high cutting speed values allows for component values of cutting force to be lowered as a result of the transition into the high-speed machining conditions range. In most cases, the highest values for the analysed parameters were recorded for the component $F_{x}$, whereas the lowest were recorded for $F_{y}$. Additionally, the paper shows that a prediction (with the use of artificial neural networks) of the components of cutting force can be made, both for the amplitudes of components of cutting force $F_{a m p}$ and for root mean square $F_{r m s}$.
\end{abstract}

Keywords High-speed dry milling $\cdot$ Cutting forces $\cdot$ Magnesium alloys $\cdot$ Entropy $\cdot$ Neural networks

\section{Introduction}

The machining allowance can be removed by means of machining methods using high cutting speeds (HSM -High Speed Machining, HPC-High Performance Cutting, HSC-High Speed Cutting), which allows for shorter machine time, increased volumetric efficiency and reduced manufacturing costs. Parts for the machine-building and aircraft industries are usually machined by milling. The socalled "utility" indicators of machinability include forces (F)

\section{Kulisz}

m.kulisz@pollub.pl

1 Department of Organisation of Enterprise, Management Faculty, Lublin University of Technology, Lublin, Poland

2 Department of Production Engineering, Mechanical Engineering Faculty, Lublin University of Technology, Lublin, Poland

3 Department of Applied Mechanics, Mechanical Engineering Faculty, Lublin University of Technology, Lublin, Poland which occur in the cutting process. A change in the cutting force components during the milling process may affect the deformation of a workpiece. [1]. In addition, changes in the cutting force may affect phenomena such as adhesion and accretion, leading to an additional decrease in surface quality, shape and dimensional accuracy. There are also studies investigating power demand in the milling of different materials, including magnesium alloys. Compared to other metals [2], the milling of magnesium alloys can be done in an effective way due to the possibility of applying high values of the technological parameters of the milling process [3], both during the square shoulder milling and roughing plunge milling process [4]. For instance, unlike in the case of Al-Si alloys, the cutting force generated in the milling of magnesium alloys is lower by approx. 50\%. Given the low cutting resistance [5] and temperature [6] in this process, the thermal and mechanical loads on the tools are low, too [7].

Cutting speed has a significant impact on the efficiency of milling processes. Given the ranges of cutting speed $v_{c}$, milling processes are often divided into those performed applying "conventional" parameters and those 
performed at higher values of $v_{c}$ (characteristic of HSM). The moment of "transition" into a range of the parameters which are typical of HSM can be defined in the following way: $\partial \mathrm{F} / \partial \mathrm{v}_{\mathrm{c}}<0$ (HSM) and $\partial \mathrm{F} / \partial \mathrm{v}_{\mathrm{c}}>0$ (conventional milling) [8]. The distinction between conventional milling and HSM is generally made when an increase in the cutting speed $v_{c}$ leads to a decrease in the cutting force. HSM is defined as a highly efficient cutting method ensuring high surface quality $(R a$ - arithmetical mean roughness of the profile parameter is often lower than $1 \mu \mathrm{m}$ ). The use of HSM helps eliminate finishing which is usually done by grinding. For instance, the use of HSM enables the shortening of milling time (by as much as four times) without decreasing surface quality and shape accuracy. The desired shape accuracy can be attained thanks to, among others, a decrease in the cutting forces in high-speed milling. Among the high-speed machining methods, one can distinguish two highly efficient methods: high performance cutting (HPC), used for pre-treatment or forming, and high-speed cutting (HSC), which is used for finishing. One recent definition of HSC has introduced the concept of so-called limit cutting speed $v_{c-g r}$ which marks the range of high-speed milling [9].

Both when the milling process is performed by tools with so-called "classical end mill geometry," as well as when a wave-shaped cutting edge is used, the cutting force components and their amplitudes are more affected by changing the feed per tooth $f_{z}$ rather than by variations in the cutting speed $v_{c}$. In the milling of AZ91HP alloy, the cutting force components are the highest when the tool with a PCD (polycrystalline diamond) end mill is used. The type of tool coating (e.g. $\mathrm{TiB}_{2}$ and $\mathrm{TiAlCN}$ ) has a significant effect on the cutting force, too. This can be particularly observed in the case of carbide end mills. The cutting force components ( $F_{x}$-component of the cutting force in the $\mathrm{x}$-axis direction, $F_{y}$-component of the cutting force in the y-axis direction) in the milling process for EN AW-6082 alloy are the lowest when a $\mathrm{TiB}_{2}$-coated tool is used. With a change in the value of $v_{c}$, one can observe a characteristic point of "transition" into an HSC machining range (at $v_{c-g r}=450 \div 600 \mathrm{~m} / \mathrm{min}$ ) [10].

Additionally, the effects of tool wear on cutting performance were investigated for milling of magnesium alloy within the cutting speed range of 1600-2000 $\mathrm{m} / \mathrm{min}$ under dry conditions in studies by [11]. These mechanisms were dominantly categorized into adhesion wear, abrasion, and diffusion under dry conditions. Extensive flaking was the significant failure mode at the cutting speed of $1600 \mathrm{~m} / \mathrm{min}$, while serious flank wear and gross fracture were mainly responsible for failures at the cutting speeds of 1800 and $2000 \mathrm{~m} / \mathrm{min}$. An increase in power consumption may also result in an increase in cutting force components and cause an increase in resistance, and as a consequence also an increase in temperature generated in the cutting zone.

In the study [12] an analysis was performed of, among others, cutting forces that were obtained during the dry face milling operation of ZE41 magnesium alloy. The variable input parameters in the process were: spindle speed, feed per tooth, depth of cut, and tool diameter. Based on studies conducted, it was observed that the cutting forces gradually increased with an increase in input parameters. These cutting forces are more closely linked with spindle speed than feed per tooth. The authors also concluded that both constant depth of cut and tool diameter are proportionally related to the cutting forces at increasing spindle speeds and feed per tooth, respectively. These forces should be kept under control as they can impact the cutting tool. When the cutting force is excessive, this also has a negative effect on the quality of a machined surface. With an increasing feed rate, the vibration in the machine-holder-workpiece-tool (MHWT) system increases, too. This is caused by the impact of excessive cutting force [13].

Based on the above, it can be seen that the values of the cutting force and of its components are influenced by many factors associated with the machining process, including but not limited to the materials from which tool is made and the material being machined, the tool geometry, and the machining parameters. Of these, however, the most significant is the selection of appropriate machining parameters, as has been demonstrated by researchers in work such as [14], while others claim that the use of high cutting speeds, feed rates, and cutting depths allows for an increase in machining efficiency. The relationships between the factors listed above are frequently nonlinear in nature. An additional drawback may be the process instability resulting from the translational motion of the end mill [15]. Moreover, during machining, at specific combinations of cutting depth and spindle speed, the regenerative effect may arise. Under chatter conditions, the vibration developed is sometimes so strong that the amplitude of the vibration between the tool and workpiece is larger than the chip thickness, so that the chip becomes dissected, and the machined surface is spoiled by chatter marks. This phenomenon is the so-called machining instability [16]. The manufacturing industry requires quality machined surfaces, and so violent chatter or machining instability must therefore be avoided.

A stability chart is commonly used to indicate stable (chatter-free) cutting as a function of spindle speed and depth of cut [17]. It has long been recognized that substantial gains in productivity can be achieved by exploiting the lobed nature of the stability chart, particularly at high speeds. The stability lobe diagram (SLD) is used in the processing of various types of materials, those commonly considered as difficult to process (titanium alloys, steel, nickel alloys), lightweight alloys (aluminium alloys, 
magensium alloys), as well as various composite materials [18]. In this paper, the authors additionally conducted statistical, recurrence, and composite multiscale entropy analyses. The authors performed a classic trial involving changing the spindle speed and observed the appearance of chatter vibrations and signatures of intermittency.

Thus, due to the complexity of the milling processes and their nonlinear nature as well as due to phenomena occurring in the cutting zone, attempts are being more and more commonly made to model the machining processes using mathematical analytical methods [4], semi-analytical techniques namely the Chebyshev collocation method and temporal finite element analysis (TFEA) [19] or using Artificial Intelligence (AI) systems [20]. Apart from Danis et al. [4], mathematical modelling was also employed by $\mathrm{Fu}$ et al. [13], and Weremczuk et al. [21]. When the nature of a process is too complex to be described in mathematical terms using equations, modelling is often performed using Neural Network Toolbox [22] or Statistica software package [20]. Artificial neural networks are most often employed for this purpose, which is confirmed in the works by Kilickap et al. [23] or Kazemi et al. [24]

The main aim of the modelling of cutting processes is the prediction of the course of the technological processes which are of a nonlinear nature. This may serve to create systems supporting decision-making processes in a business, for example when selecting technological machining parameters. For the modelling and simulation of cutting force components, neural networks can be used, such as RBF (Radial Basis Function) and MLP (Multi-Layered Perceptron). The authors of one study [25] presented a model for the prediction of components of cutting force for an AZ91HP alloy with two inputs, namely the machining parameters (cutting speed and feed per tooth). Other materials which have been studied by other researchers using simulations of cutting force components include aluminium alloy (input parameters: spindle speed, feed per tool, radial depth and axial depth) [26], 7050 aluminium alloy (input parameters: spindle speed, feed per tooth, milling width) [27], Inconel 718 (input parameters: tool wear, feed per tooth, cutting depth, cutting width, cutting speed) [28], and also AZ31 magnesium alloys with a PCD tool (cutting speed range and feed per tooth as input parameters) [10]. The models developed allow for the testing of different configurations of input parameters (machining parameters) without having to simultaneously conduct experimental tests. The results of our experiments show that the measured results and simulated results correspond well with each other. Modelling and simulation can also be used to predict the influence of technological parameters on other indicators, e.g. the surface quality [29] achieved by machining processes.

Additionally, it is becoming increasingly common in the analysis of dynamic processes to use the multiscale entropy method (MSE), defined as a measure of the degree of disorder or uncertainty of a given signal, allowing for an assessment of the complexity of time series. This is based on a granulation procedure conducted by averaging original points on the time series [30]. Unfortunately, with a large scale factor $\tau$, the results may be skewed by an error which can be eliminated by applying the concept of composite multiscale entropy (CMSE) [31].

The aim of this paper is to analyse the impact of changes in technological parameters on the cutting force values in the case of milling an AZ31 magnesium alloy with two different tools. This alloy is relatively rarely analysed in milling processing, to a much lesser extent than the AZ91D magnesium alloy. Carrying out investigations for two types of cutting tools can reveal some differences in the course of milling process. analysis made use of courses of cutting forces obtained experimentally, based on which a statistical analysis was conducted. The study also made use of composite multiscale entropy (CMSE) as a measure of disorder in the time series of the cutting force signal. It is believed that it can be an extension of the analyses conducted on the basis of commonly used indicators and provide additional information on the course of the cutting process. Additionally, the use of neural networks was proposed to develop predictive models for the component values of total cutting force, and the values of the amplitudes of cutting forces $F_{a m p}$ and root mean square $F_{r m s}$ for the TiAlN-coated tool. The input parameters for modelling were the technological parameters of the process (cutting speed and feed per tooth). An analysis of the literature shows that no such modelling has been so far conducted.

\section{Experimental set-up}

The main aim of the study was to investigate the effect of parameters such as cutting speed $v_{c}$ and feed per tooth $f_{z}$ on the dynamics of a system during milling. The experimental investigations were conducted on AZ31 magnesium alloy specimens cut on an AVIA VMC 800HS machining centre. Chemical composition and mechanical properties of the machined magnesium alloy are shown in the Table 1.

The tools used in the study were $16 \mathrm{~mm}$ end mills $(z=2)$, one carbide TiAlN-coated (Fenes) and the other with a PCD end mill (Guhring). On the basis of the conducted literature review, it was observed that these tools enable achieving favourable machining effects during the milling of magnesium alloys. These tools are frequently employed in milling of various lightweight alloys, a carbide TiAlN-coated tool is relatively cheap and yields good machining effects, whereas a PCD tool enables to achieve high quality of the surface, comparable to grinding $\mathrm{Ra} \leq 0.16 \mu \mathrm{m}$ (elimination of additional machining processes). A constant radial depth 
Table 1 Chemical composition and mechanical properties of AZ31 magnesium alloy [32]

\begin{tabular}{|c|c|c|c|c|c|}
\hline \multicolumn{6}{|c|}{ Chemical composition } \\
\hline $\mathrm{Al}$ & Mn & $\mathrm{Zn}$ & $\mathrm{Cu}$ & Others & $\mathrm{Mg}$ \\
\hline $2.5-3.5$ & $0.1-0.2$ & $0.7-1.3$ & 0.05 & 0.04 & Rest \\
\hline \multicolumn{6}{|c|}{ Mechanical properties } \\
\hline $\mathrm{R}_{\mathrm{m}}(\mathrm{MPa})$ & $\mathrm{R}_{\mathrm{p} 0.2}(\mathrm{MPa})$ & $\mathrm{E}(\mathrm{GPa})$ & $\mathrm{A}_{5}(\%)$ & $\mathrm{HB}$ & $\rho\left(\mathrm{g} / \mathrm{cm}^{3}\right)$ \\
\hline $250-290$ & $150-220$ & $\sim 45$ & $12-21$ & $46-73$ & 1.78 \\
\hline
\end{tabular}

of cut $a_{e}=14 \mathrm{~mm}$, axial depth of cut $a_{p}=6 \mathrm{~mm}$, and the following technological parameter ranges were applied: $v_{c}=200-1200 \mathrm{~m} / \mathrm{min}$ and $f_{z}=0.05-0.30 \mathrm{~mm} /$ tooth, as shown in Table 2. The technological parameter ranges $v_{c}$ and $f_{z}$ were selected on the basis of the previous studies on magnesium alloys, which can be found in other available publications as well as on the basis of the previous own studies and literature review. In addition, $v_{c}$ was obtained via studies and analyses of HSM; however, it is limited by the technological capacity of the machining device (maximum spindle speed of 24,000 rpm).

Figure 1 shows a plan of the experiments and a schematic diagram of the experimental set-up. The experimental setup is composed of two subsystems: a modal analysis system and a dynamometer system. The former is used to measure tool-holder stiffness and damping coefficient (modal parameters). It consists of the modal hammer PCB 086C03, accelerometer PCB 352B10, data acquisition card (DAQ) NI9234 and CutPro software. The latter is used to measure the cutting force components $\left(F_{x}, F_{y}\right.$ and $\left.F_{z}\right)$ by means of a Kistler 9257B piezoelectric dynamometer connected to a Kistler 5017B signal conditioner and a SCADAS Mobile LMS analyser. Both experimental rigs are integrated in the computer system. Measurements are conducted in two steps. In the first, the impact test is done to get data for the stability lobe diagram (SLD). The modal hammer is used to excite the tool and then the resulting vibrations are measured by the low mass accelerometer mounted at the tool tip. Next, the modal parameters in the form of frequency response function (FRF) are implemented to CutPro9 software, which calculates and plots SLD for three different values of feed per tooth $f_{z}$ (Fig. 2). In the second step of the experiment, a verification of unstable lobes is performed for series of spindle speeds and the depths of cut presented as black points in the SLD. Experimental verification of stability lobes is frequently employed during the experimental studies of the machining processes. The parameters, based on which the CutPro software determines the stability curves, are static. However, these parameters may change during processing and thus change the location of stability lobes.

SLD was prepared only for a carbide TiAlN-coated tool. In the case of PCD tool, creation of SLD would be risky, because a relatively brittle part of the tool made of polycrystalline diamond should be struck with a modal hammer, which could result in its damage.

The location of SLD curves affects the rigidity and vibration damping of the system. Diameter and free length of the tool were the same in both cases; thus, the system rigidity did not change. The influence of blade shape and the type of coating affect the MHWT system rigidity to a minimal
Table 2 Technological parameters with which the milling process was carried out

\begin{tabular}{cllll}
\hline Cutting tool & $\begin{array}{l}\text { Cutting speed } \\
v_{c}(\mathrm{~m} / \mathrm{min})\end{array}$ & $\begin{array}{l}\text { Feed per tooth } \\
f_{z}(\mathrm{~mm} / \text { tooth })\end{array}$ & Axial depth of cut $a_{p}(\mathrm{~mm})$ & Radial depth of cut $a_{e}(\mathrm{~mm})$ \\
\hline PCD, TiAlN & 200 & 0.15 & 6 & 14 \\
400 & & & \\
600 & & & \\
800 & & & \\
1000 & & & \\
1200 & 0.05 & \\
800 & 0.10 & \\
& 0.15 & \\
& 0.20 & \\
& 0.25 & \\
& 0.30 &
\end{tabular}




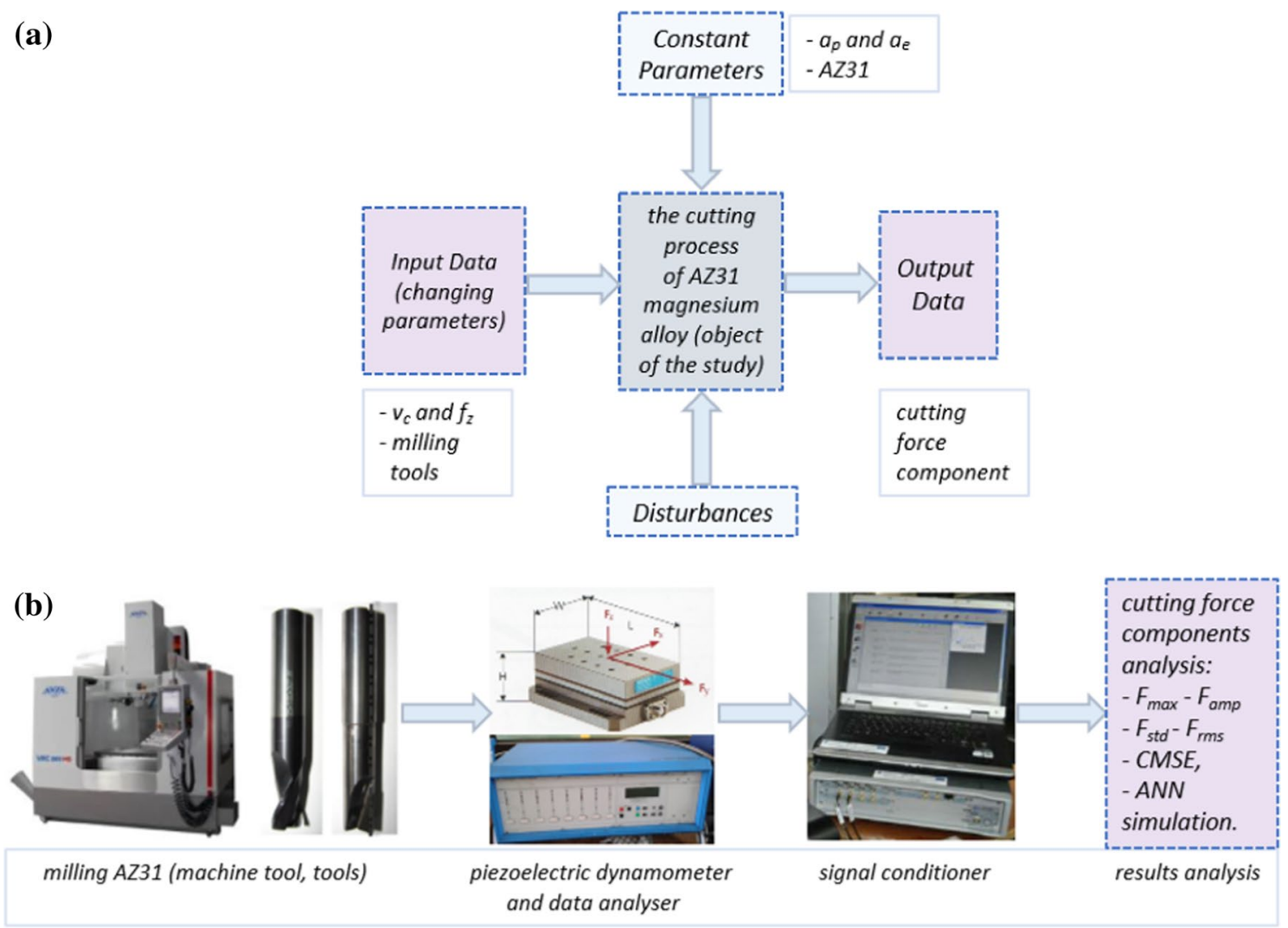

(c)

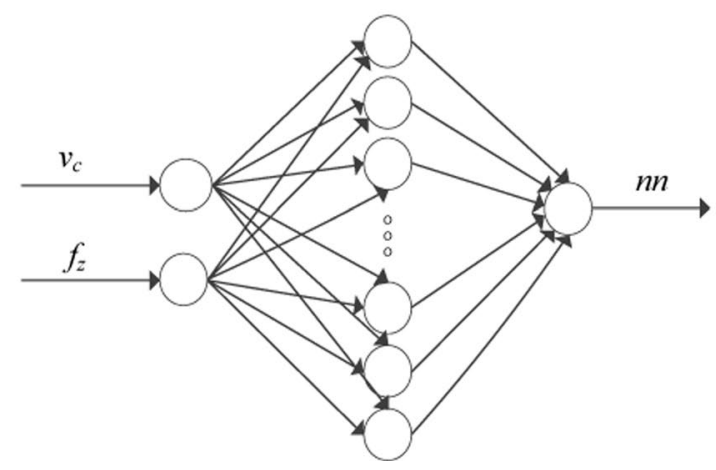

Fig. 1 Design of the: $\mathbf{a}$ experiment and $\mathbf{b}$ schematic diagram of the experimental set-up $\mathbf{c}$ schematics of the artificial neural network with the analysed process parameters

degree. Thus, it can be concluded and assumed with high degree of probability that the SLD curves are similar for both tools (or even identical).

The cutting forces generated on the workpiece during the machining were measured by a dynamometer mounted on the milling machine underneath the workpiece. The corresponding force signals from the dynamometer were first transmitted to the charge amplifier, next to the analyser and, finally, to the computer system. The sampling rate of data recorded during the test was set to $1 \mathrm{kHz}$.

A typical, experimentally obtained time series is presented in Fig. 3. In the paper, the maximum value $F_{\max }$ is the highest positive value of the signal. The $F_{a m p}$ amplitude is the highest deviation from equilibrium. Higher values $F_{\text {amp }}$ than $F_{\max }$ result from the signal asymmetry (shifted towards the bottom). In the presented case, $F_{\text {max }}$ is approximately equal to $400 \mathrm{~N}$, while $F_{a m p}$ is about $900 \mathrm{~N}$.

An additional aim of this study was to verify whether it is possible to predict the impact of technological parameters $\left(f_{z}\right.$ and $v_{c}$ ) on the maximum values of the components of total cutting force and on the values of the amplitudes of cutting forces $F_{a m p}$ and the root mean square $F_{r m s}$ when rough milling an AZ31 magnesium alloy using a carbide TiAlNcoated tool. Thus, a simulation of selected components of 


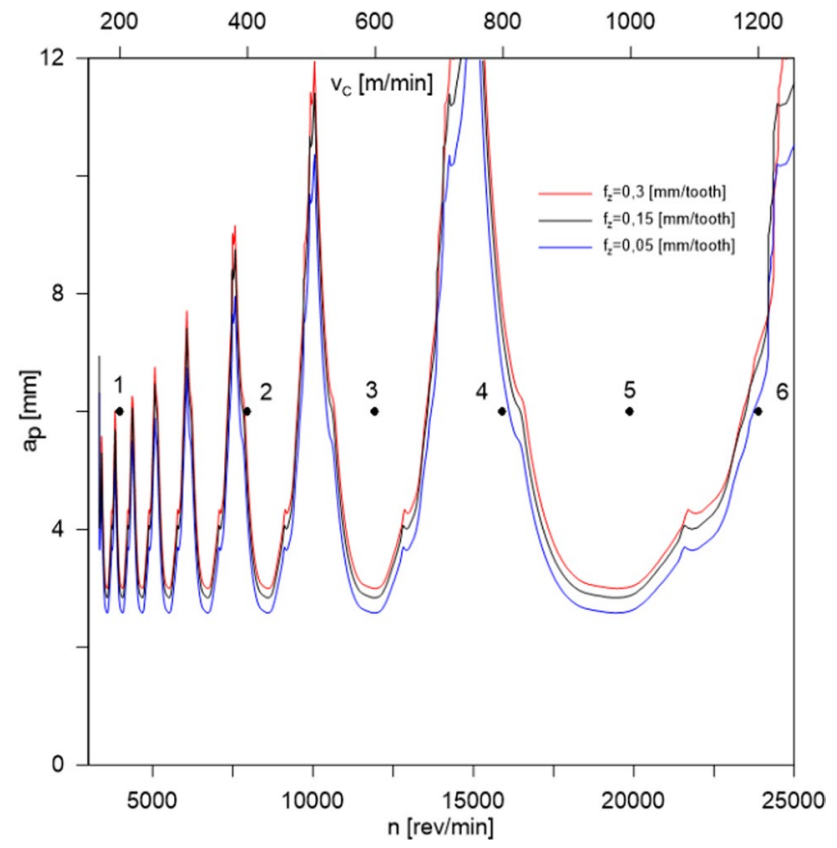

Fig. 2 Stability lobe diagram for milling processes using a carbide TiAlN-coated (Fenes) tool

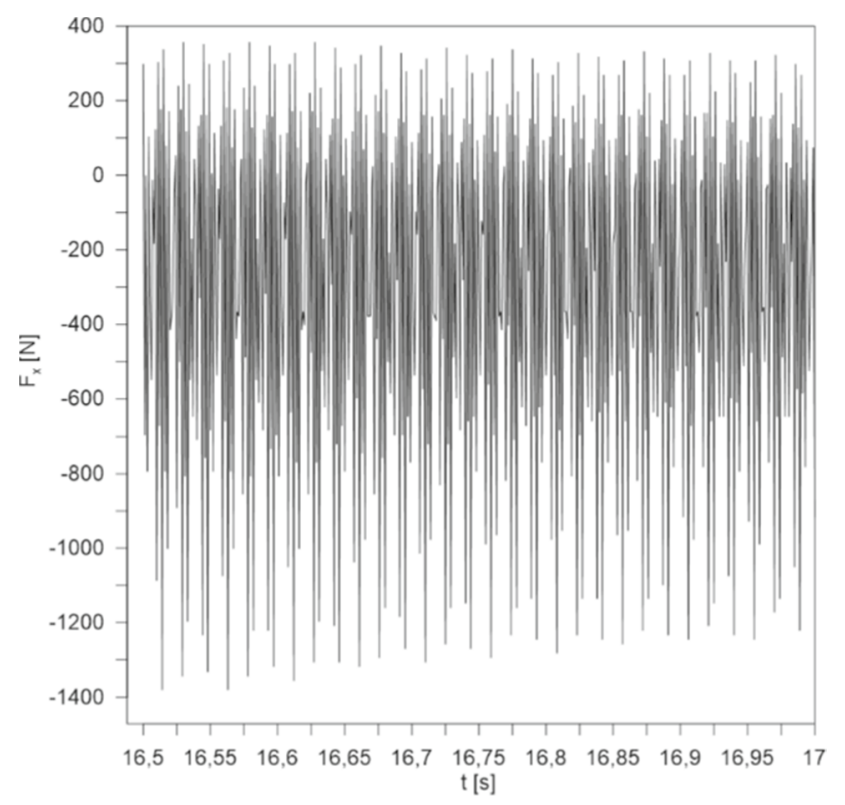

Fig. 3 Time series for PCD tool $\left(f_{z}=0.05 \mathrm{~mm} /\right.$ tooth $)$

cutting force $\left(F_{x}\right.$ and $\left.F_{y}\right)$ and amplitude of the component $F_{x}$ as well as the root mean square for the component $F_{x}$ was conducted. The component $F_{x}$ was selected because it has the greatest impact on the total cutting force.

To conduct the simulation, the Statistica 13 Neural Networks software package was used, and input data comprised the results of experimental studies. The input neurons for training the network were the machining parameters $v_{c}=200-1200 \mathrm{~m} / \mathrm{min}$ and $f_{z}=0.05-0.30 \mathrm{~mm} /$ tooth, while the output neuron for modelling a given networks was the cutting force component/amplitude of component $F_{x} /$ root mean square of the component $F_{x}$. Therefore, modelling was performed for 4 different networks. Two types of network were used for modelling, an MLP (multi-layered Perceptron) network and an RBF (Radial Basis Function) network. The set of training and verification data was divided in a proportion of $75 \%$ (training set) to $25 \%$ (verification set). The test data set was omitted due to the small number of input data for training the network [21].

With the MLP network, linear, logistic, exponential, tanh and sinus activation functions were applied, while with the RBF network, Gaussian (hidden neurons) and linear (output neurons) functions were applied. The networks were modelled with a single layer of hidden neurons within a range of $2-9$. The number of epochs fluctuated from 150 to 500. For each model, 100 networks were constructed and the best of these was selected based on training and verification quality as well as on errors in these sets. Errors were calculated using the least squares method.

\section{Experimental test results}

In the following study, the statistical analysis waveform forces were produced by experimental investigations. The bar charts show the results of the forces in three directions for two different tools (a PCD and a TiAlN-coated tool). The tests were carried out for six parameters, and are shown in the stability lobe diagram as points from 1 to 6 (Fig. 2).

\subsection{Influence of cutting speed}

First, the impact of the cutting speed $v_{c}$ was investigated. Figure 4a shows the change in the maximum cutting forces $F_{\text {max }}$ in relation to the cutting speed. In the case of both tools, the highest values of maximum forces $F_{\max }$ were observed in the $x$ direction (feed direction), the smallest in the $z$ direction. Similar relations are visible when analysing the amplitudes of cutting forces $F_{\text {amp }}$ (Fig. 4b). In the case of cutting speed $v_{c}=800 \mathrm{~m} / \mathrm{min}$, a significant difference in the value of the cutting force amplitudes $F_{a m p}$ was observed for both tools in the feed direction. The highest values of standard deviations $F_{\text {std }}$ (Fig. 5b) and root mean square $F_{r m s}$ (Fig. 5a) were observed at the cutting speed of $v_{c}=600 \mathrm{~m} / \mathrm{min}$.

From the experimental tests carried out for the AZ31 alloy, it can be concluded that with the increase of the cutting speed $v_{c}$ to $600 \mathrm{~m} / \mathrm{min}$, all the components of the cutting forces increase (most components in the feed direction). At the same time, above $v_{c}=800 \mathrm{~m} / \mathrm{min}$ a significant reduction in cutting forces was observed for both tools. 
(a)

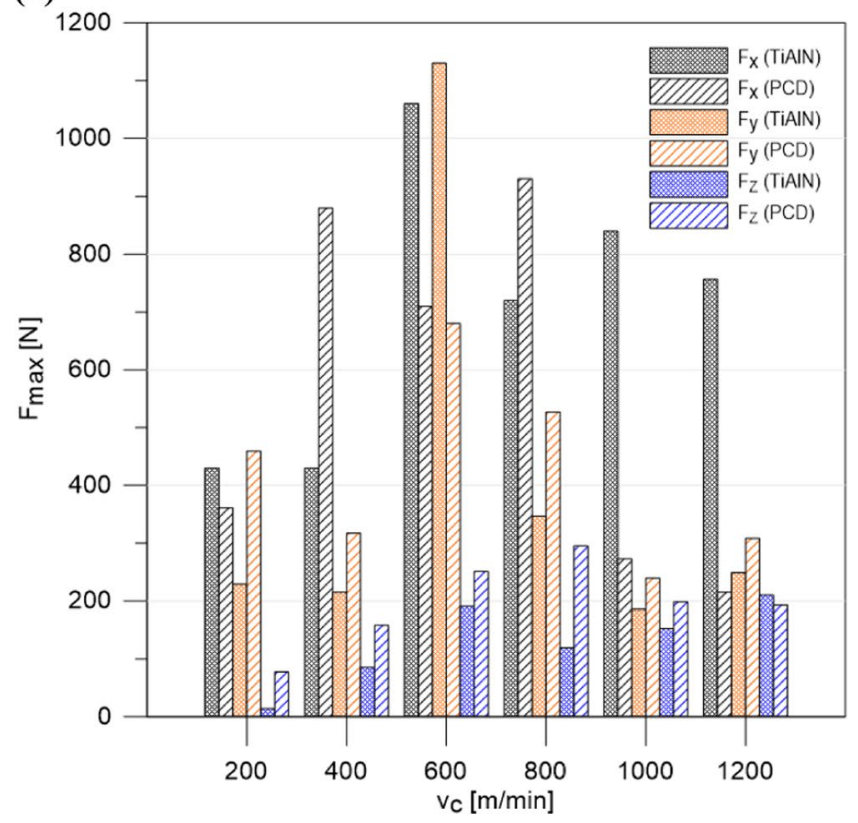

(b)

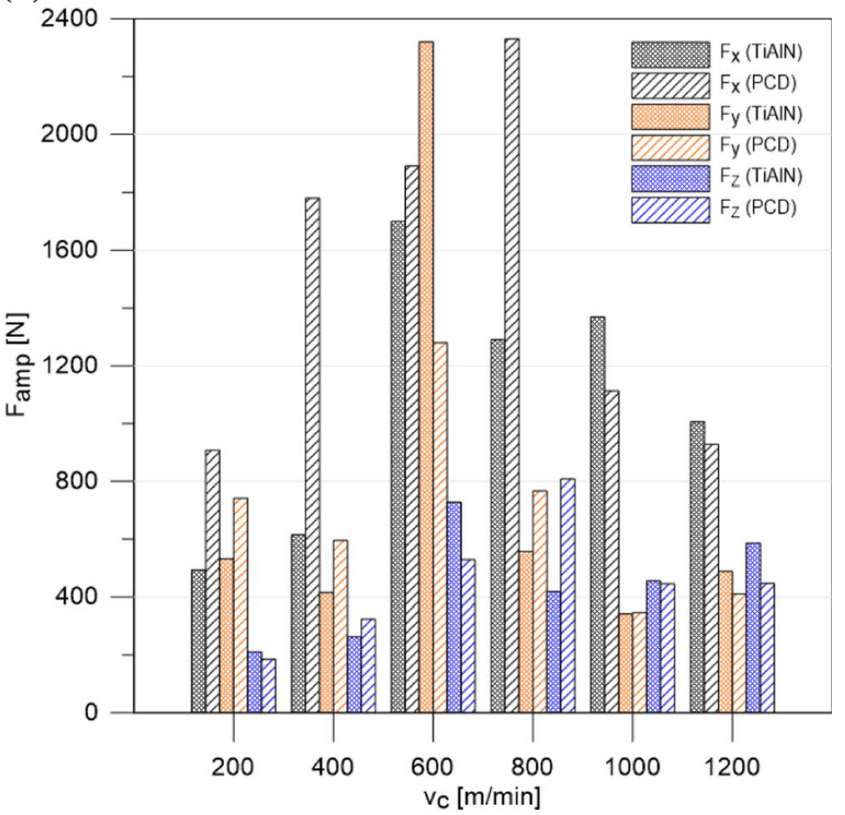

Fig. 4 The effect of change in cutting speed $v_{c}$ on the value of cutting forces for $f_{z}=0.15 \mathrm{~mm} /$ tooth a maximum $F_{\max }$ and $\mathbf{b}$ amplitude $F_{\text {amp }}$ in the milling of AZ31 magnesium alloys using different tools

(a)

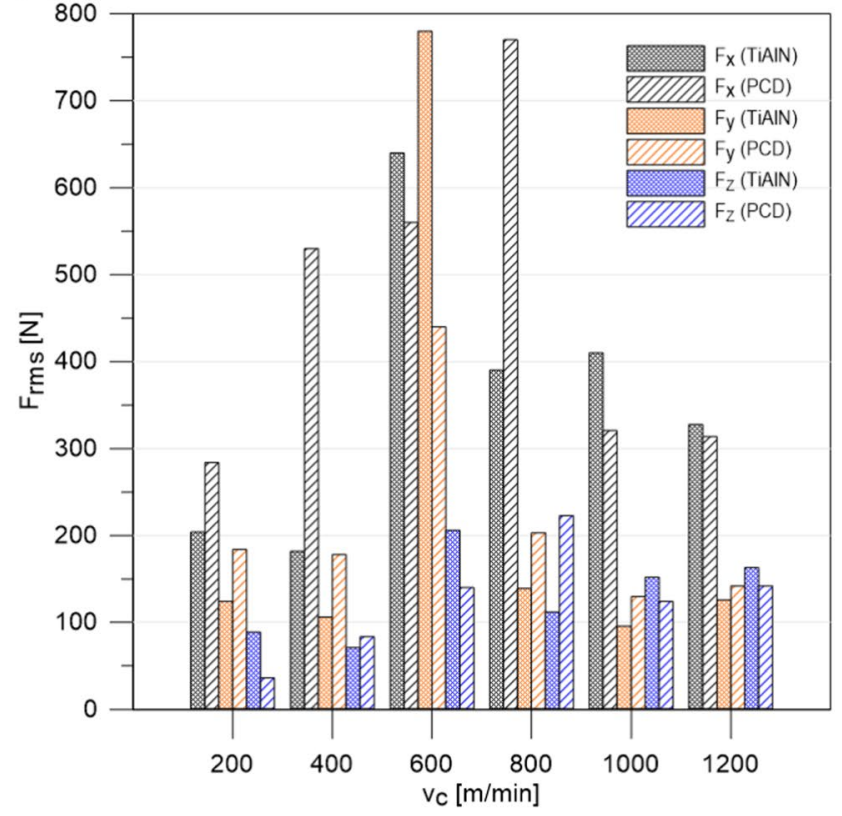

(b)

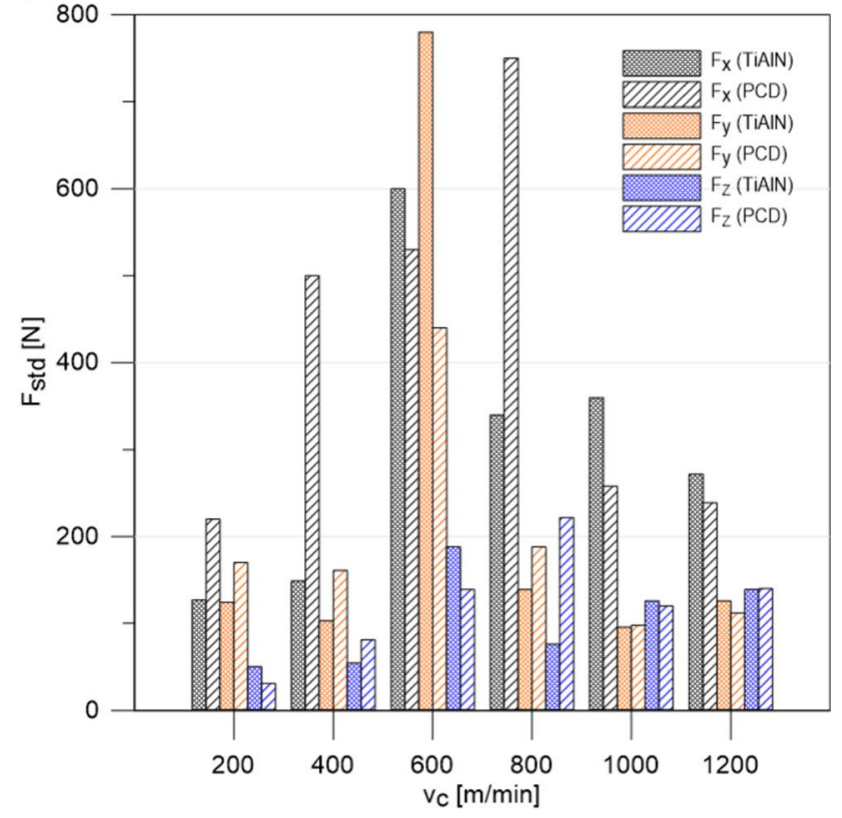

Fig. 5 The effect of change in cutting speed $v_{c}$ on the value of cutting forces for $f_{z}=0.15 \mathrm{~mm} /$ tooth a root mean square $F_{r m s}$ and $\mathbf{b}$ standard deviation $F_{\text {std }}$ in the milling of AZ31 magnesium alloys using different tools

This is due to the characteristic transition to HSM machining for higher cutting speeds. Significantly, lower maximum cutting forces $F_{\max }$ were observed when cutting using the PCD tool. However, when analysing the amplitude of cutting forces $F_{a m p}$, smaller values were obtained using the TiAlN-coated tool. In the case of effective value $F_{r m s}$ and standard deviation $F_{\text {std }}$, the highest values were again obtained for the component $F_{x}$. The lowest values of indicators when milling at low cutting speeds were noted for 
component $F_{z}$, though after achieving a cutting speed of $v_{c}=800 \mathrm{~m} / \mathrm{min}$ the lowest values were seen for $F_{y}$.

The dramatic increases in values which are repeated in the case of all analysed indicators, particularly visible for the cutting speed range of $v_{c}=400-800 \mathrm{~m} / \mathrm{min}$, can be associated with the course of stability curves. Rotational speeds equivalent to cutting speeds of $v_{c}=400 \mathrm{~m} / \mathrm{min}$ and $v_{c}=800 \mathrm{~m} / \mathrm{min}$ were determined based on stability curves to be speeds which generated instability during the machining process. Despite the fact that the machining process was conducted with all remaining values of technological parameters within the unstable machining zones of the stability curves, clear increases in these values were not observed. This may be associated with the feed per tooth value of $f_{z}=0.15 \mathrm{~mm} /$ tooth which the sample millings were conducted at, as the stability curves were generated for feed per tooth of $f_{z}=0.05 \mathrm{~mm} /$ tooth and $f_{z}=0.30 \mathrm{~mm} /$ tooth.

\subsection{Influence of feed per tooth}

In the next step, the influence of the feed per tooth $f_{z}$ was investigated. The change of the feed per tooth was carried out in the range $f_{z}=0.05-0.30 \mathrm{~mm} /$ tooth in six steps. Together with the increase of the feed per tooth, all components of the cutting forces increase; this is visible for the $F_{x}$ component (feed direction) for both tools (Fig. 6 and Fig. 7).

In the case of cutting with the TiAlN-coated tool, higher values of maximum forces $F_{\max }$ for the $F_{x}$ component were observed. However, observing the other indicators, it can be concluded that the waveforms obtained during cutting with the PCD tool show greater variability. This is evidenced by the much higher $F_{a m p}$ (Fig. 6b), $F_{r m s}$ (Fig. 7a) and $F_{\text {std }}$ (Fig. $7 \mathrm{~b}$ ) values for the $F_{x}$ component in the case of the PCD tool.

The use of a carbide tool with a TiAlN coating provides greater stability of the milling process at a wide range of feed per tooth. As in the case of changes in cutting speed, the highest values of the analysed indicators were noted for component $F_{x}$, while the lowest were noted for $F_{z}$.

\section{Multiscale entropy analysis}

In further analysis of the cutting forces, the composite multiscale entropy (CMSE) method was used [31]. In this method, $\tau$ is the scale factor. The original time series is divided into non-overlapping windows of length $\tau$ and the data points inside each window are averaged.

$y_{k, j}^{(\tau)}=\frac{1}{\tau} \sum_{i=(j-1) \tau+k}^{i=j \tau+k-1} x_{i}, 1 \leq j \leq \frac{N}{\tau}, 1 \leq k \leq \tau$,

where $N$-length of data series, $x$ - time series.

At each scale factor $\tau$, the composite multiscale entropy calculation is based on the averaged time series $\{y\}$ and reads as

(b)

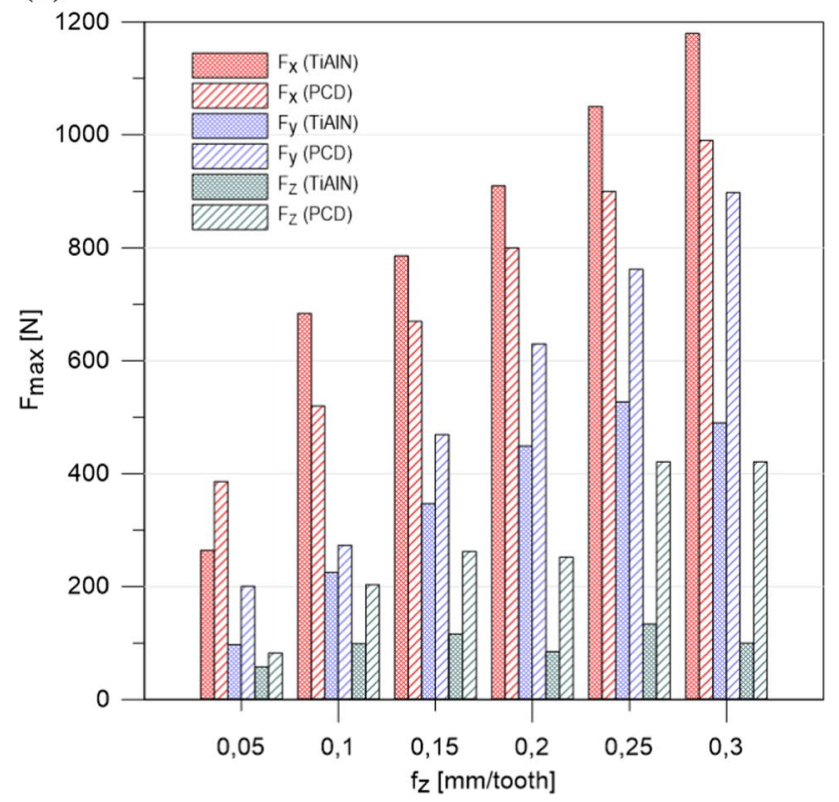

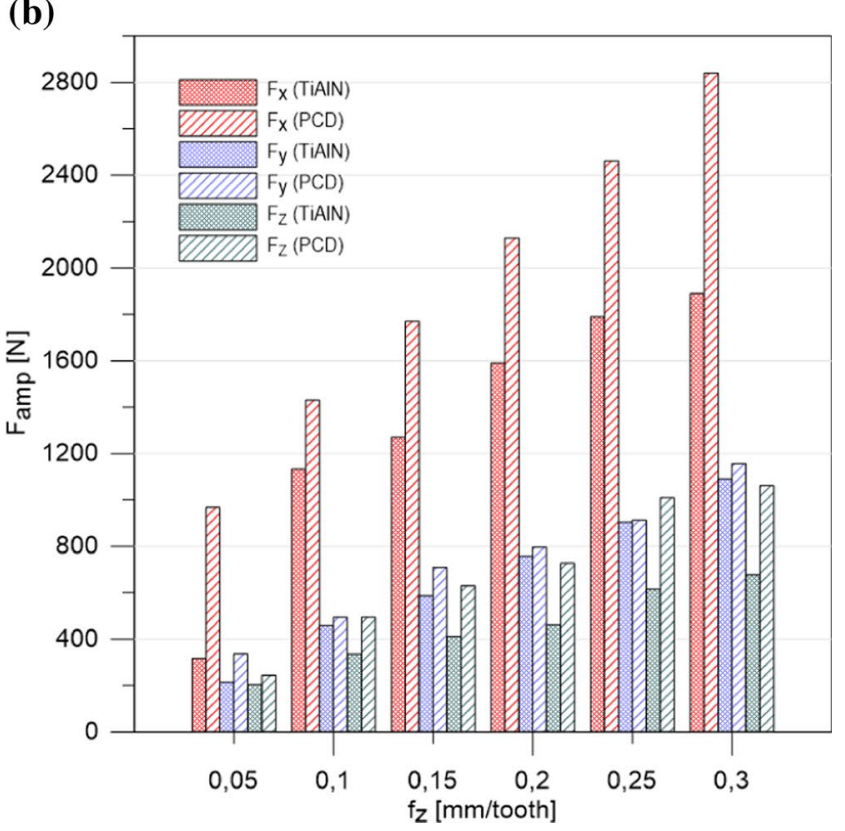

Fig. 6 The effect of change in feed per tooth $f_{z}$ on the value of cutting forces for $v_{c}=800 \mathrm{~m} / \mathrm{min}$ a maximum $F_{\text {max }}$ and $\mathbf{b}$ amplitude $F_{\text {amp }}$ in the milling of AZ31 magnesium alloys using different tools 
(a)

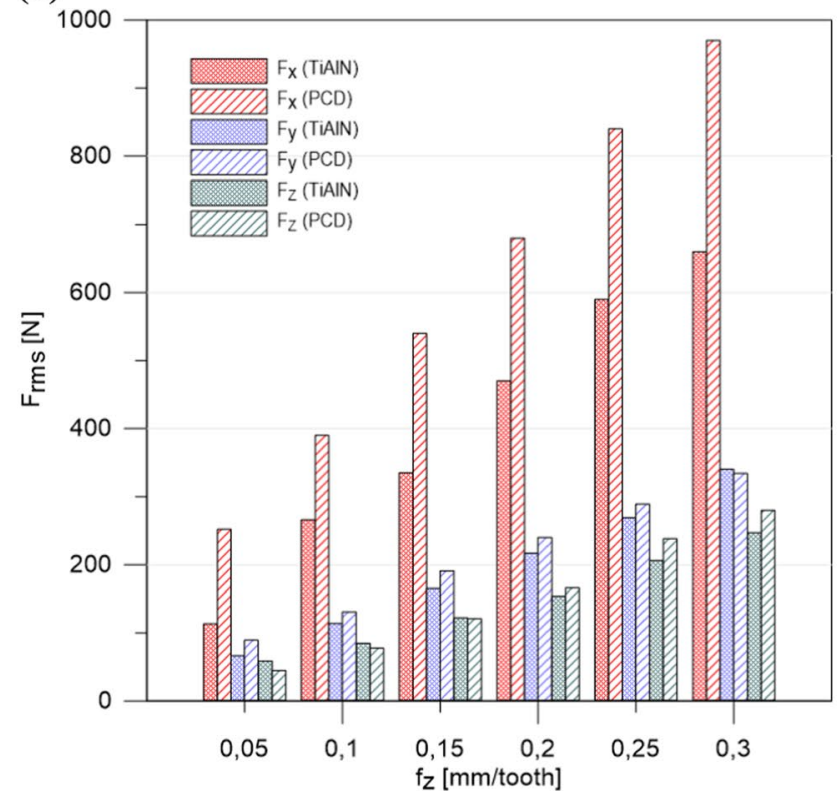

(b)

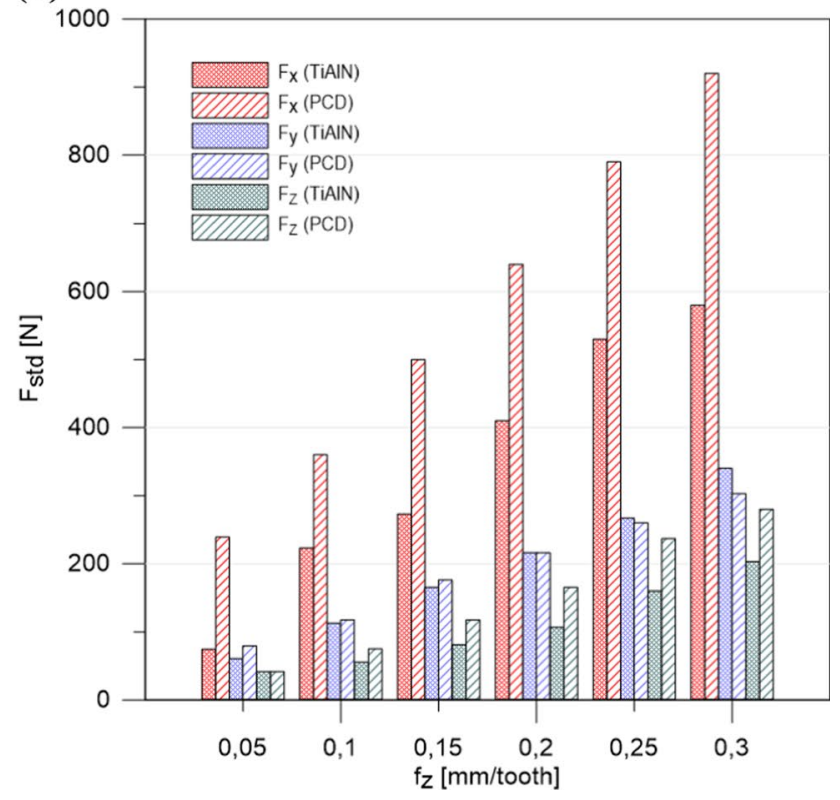

Fig. 7 The effect of change in feed per tooth $f_{z}$ on the value of cutting forces for $v_{c}=800 \mathrm{~m} / \mathrm{min}$ a root mean square $F_{r m s}$ and $\mathbf{b}$ standard deviation $F_{\text {std }}$ in the milling of AZ31 magnesium alloys using different tools

(a)

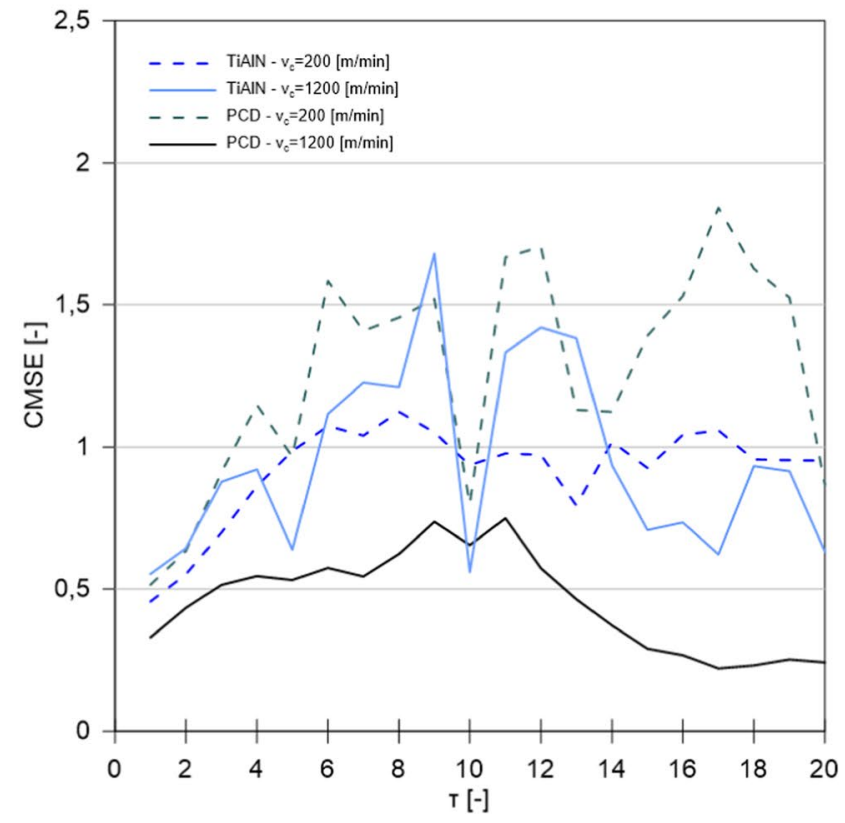

(b)

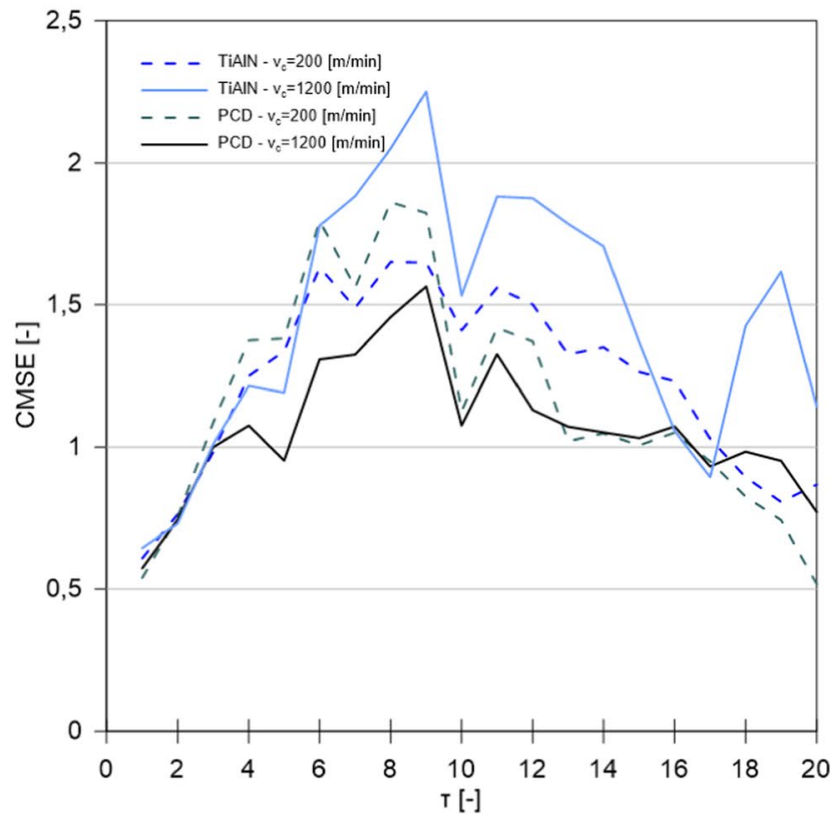

Fig. 8 The composite multiscale entropy analysis for measured cutting force components $\mathbf{a} F_{x}$ and $\mathbf{b} F_{y}$ signals for two different values of the cutting speed and $f_{z}=0.15 \mathrm{~mm} /$ tooth

$\operatorname{CMSE}(x, \tau, m, r)=\frac{1}{\tau} \sum_{k=1}^{\tau} \operatorname{SampEn}\left(y_{k}^{(\tau)}, m, r\right)$, where $m=2$ is the pattern length and $r$ is the similarity criterion, which is usually equal to $10 \%$ of the standard deviation of the original time series $\{x\}$ used. 

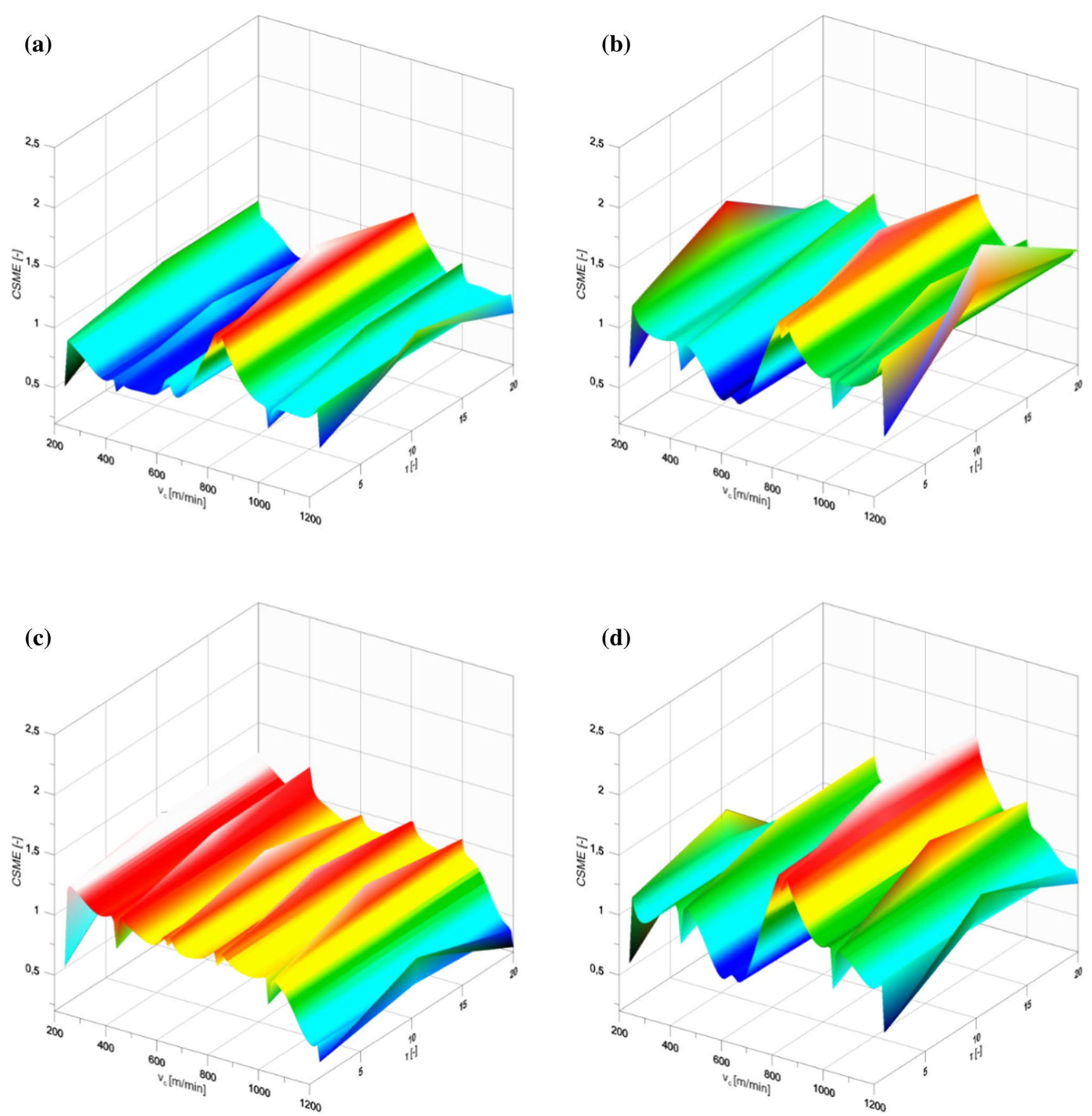

Fig. 9 The maps of composite multiscale entropy against cutting speed and scale factor $\tau$ at $f_{z}=0.15 \mathrm{~mm} /$ tooth for the TiAlN-coated tool a $F_{x}, \mathbf{b}$ $F_{y}$ and for PCD tool $\mathbf{c} F_{x}, \mathbf{d} F_{y}$

Next, the method of composite multiscale entropy was used to analyse the experimental cutting forces. Figure 8 shows the composite multiscale entropy for two values of cutting speed $\left(v_{c}=200 \mathrm{~m} / \mathrm{min}\right.$ and $v_{c}=1200 \mathrm{~m} / \mathrm{min}-$ points 1 and 6 on Fig. 2) and two different tools. In the case of component $F_{x}$, a considerable increase in entropy was observed for milling using the PCD tool at a speed of $v_{c}=200 \mathrm{~m} / \mathrm{min}$ (Fig. 8a). Change in speed only slightly impacted the change in the level of entropy when using the TiAlN-coated tool. Similar behaviour was observed for the entropy levels of component $F_{y}$ (Fig. 8b).

The change in multiscale entropy of the component signals $F_{x}$ and $F_{y}$ dependent on the change in cutting speed is also presented in the form of maps for the TiAlN-coated tool (Fig. 9a, b) and the PCD tool (Fig. 9c, d). The highest levels of entropy when milling with the TiAlN-coated 
(a)

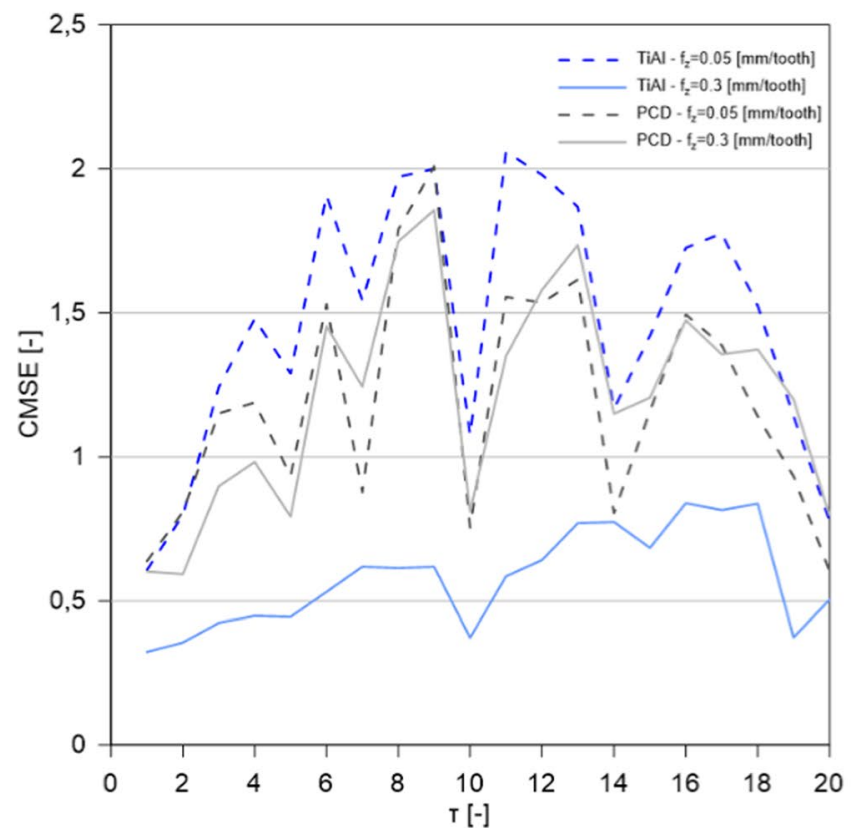

(b)

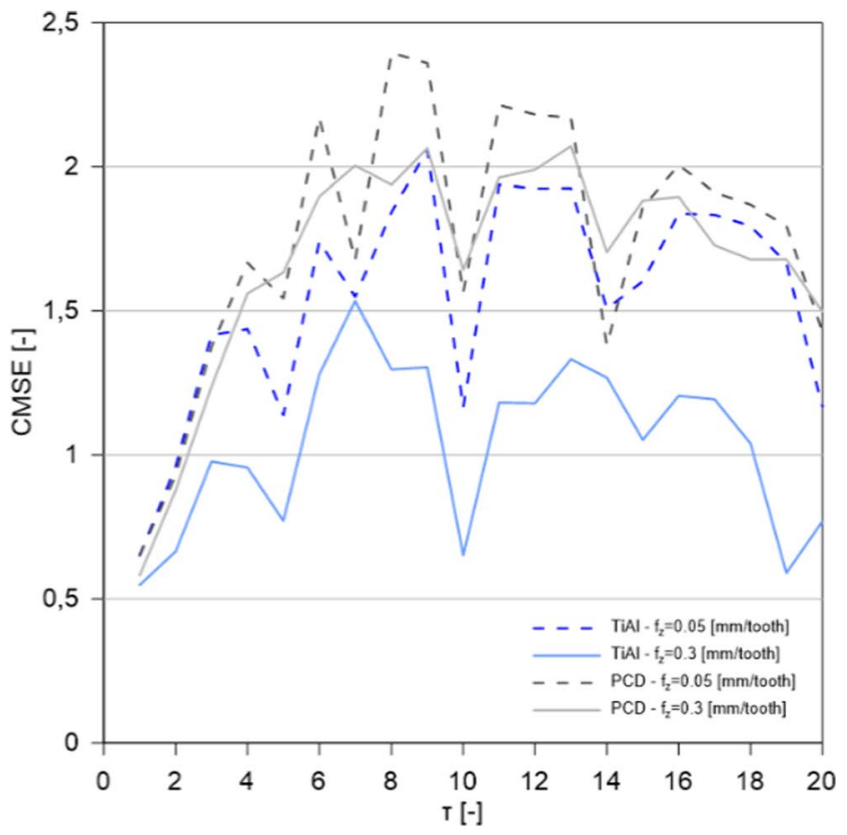

Fig. 10 The composite multiscale entropy analysis for measured cutting force components $\mathbf{a} F_{x}$ and $\mathbf{b} F_{y}$ signals for two different values of feed per tooth and $v_{c}=800 \mathrm{~m} / \mathrm{min}$

tool occurred for cutting speed of $v_{c}=800 \mathrm{~m} / \mathrm{min}$, which coincide with dramatic rises in the values of cutting force components and which may be the result of machining in the unstable SLD area. In the case of component $F_{y}$, the level of entropy increases for successive values of the scale factor $\tau$, and this effect subsides along with an increase in cutting speed. The level of entropy for component $F_{y}$ is characterized by considerably greater variability. This effect occurs regardless of the type of cutting tool used.

Figure 10 shows the composite multiscale entropy for two values of feed per tooth $\left(f_{z}=0.05 \mathrm{~mm} /\right.$ tooth and $f_{z}=0.30 \mathrm{~mm} /$ tooth) and two different tools. In the case of feed direction (Fig. 10a), it was observed that for the feed $f_{z}=0.30 \mathrm{~mm} /$ tooth, the value of entropy is reduced. Significantly lower values are observed when cutting with the TiAlN coating tool. Similar results were observed in the $y$ direction (Fig. 10b), but the value of entropy was higher than the $x$ direction. Moreover, the difference in the obtained values (Frms/Fstd and entropy) confirms that the application of an additional index, i.e. composite multiscale entropy enables observing the process from a different point of view.

A map of changes in multiscale entropy of component signals $F_{x}$ and $F_{y}$ for the TiAlN-coated tool (Fig. 11a, b) and PCD tool (Fig. 11c, d), also presented in relation to changes in feed per tooth.

In the case of component $F_{x}$, the course of entropy displays considerable variability, while for component $F_{y}$ the level of entropy in the initial range of the scale coefficient increases, then gradually decreases, particularly in the case of the PCD tool. The character of changes in entropy is maintained for both tools throughout the full range of changes in feed per tooth. A decrease in the level of entropy of both cutting force components can only be seen when milling with the TiAlN-coated tool at the highest feed per tooth of $f_{z}=0.30 \mathrm{~mm} /$ tooth.

\section{Simulation results}

Based on the results of the modelling process conducted for selected maximum cutting force components $\left(F_{x}\right.$ and $F_{y}$ ), amplitude of component $F_{x}$, and root mean square for component $F_{x}$, for each model a network with the best indicators was selected, for which the quality of training and verification was the highest, and for which the errors in training and verification were the lowest. The characteristics of these networks are described in Table 3. The best results were obtained for component $F_{x}$ for the network MLP 2-2-1 with two neurons obtained in 315 iterations, while for component $F_{y}$ the best results were obtained for a network with six neurons (MLP 2-6-1) obtained in 438 iterations. In the case of modelling of the amplitude of cutting force component $\mathrm{F}_{\mathrm{x}}$, the best network was obtained in 356 iterations, having 9 neurons in the hidden layer (MLP 2-9-1). For these three models, the best models were obtained for the MLP network, while only in the case of 

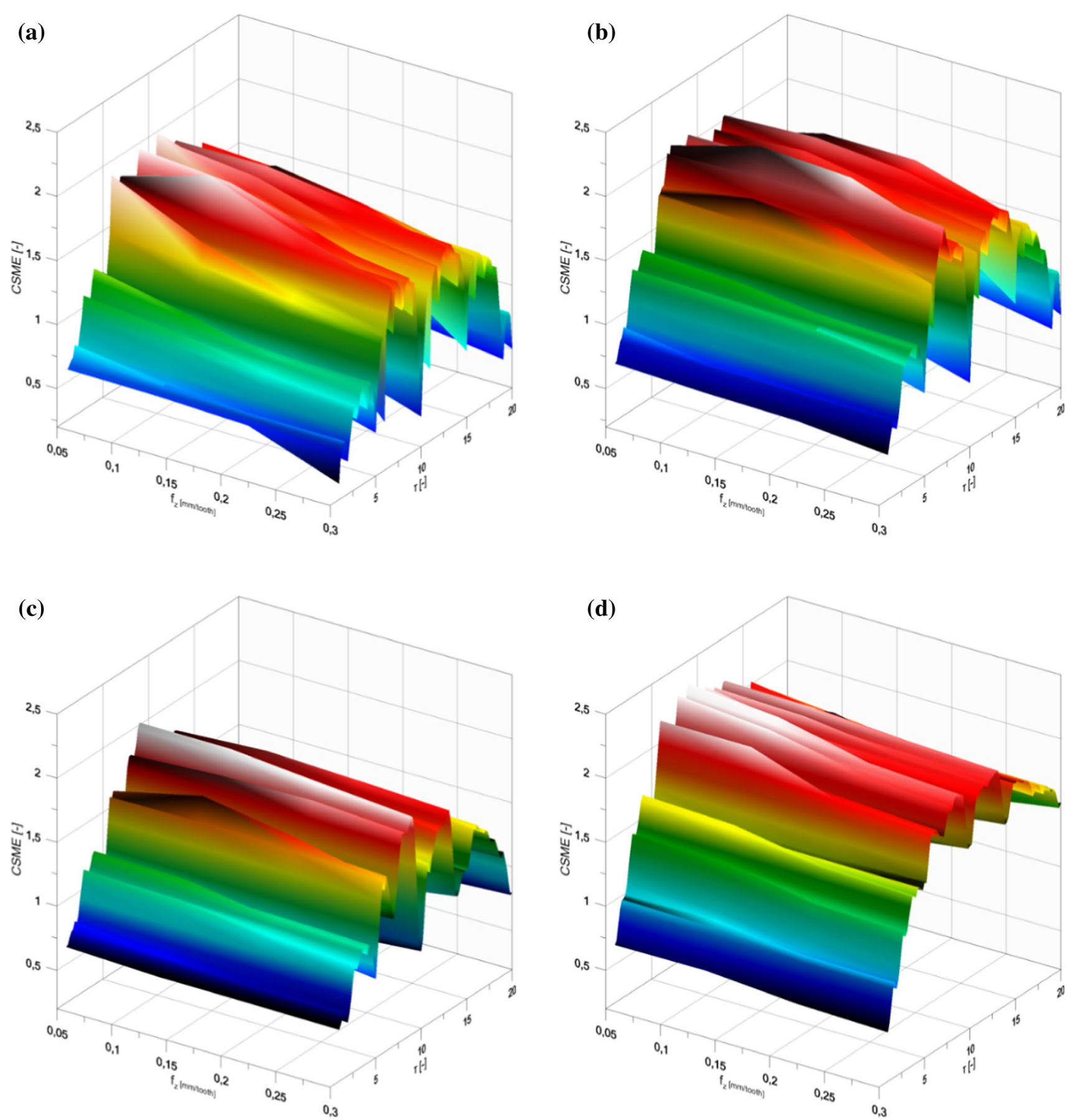

Fig. 11 Maps of composite multiscale entropy against of feed per tooth and scale factor $\tau$ at $v_{c}=800 \mathrm{~m} / \mathrm{min}$ for TiAlN-coated tool a $F_{x}, \mathbf{b} F_{y}$ and for PCD tool $\mathbf{c} F_{x}, \mathbf{d} F_{y}$

root mean square of cutting force component $\mathrm{F}_{\mathrm{x}}$ were better parameters obtained for the RBF 2-9-1 network.

The numerical results for the modelled parameters are presented below. Figure 12a) presents results for the cutting force component $F_{x}$, Fig. 12b) for cutting force component $F_{y}$, Fig. 12c for the amplitude of cutting force component $F_{x}$, and Fig. 12d) for the root mean square of cutting force component $F_{x}$. These are the results of a simulation obtained after entering the assumed input parameters $v_{c}, f_{z}$ for each of the generated networks. 
Table 3 Characteristics of selected networks: cutting force components $\left(F_{x}\right.$ and $\left.F_{y}\right)$, amplitude of component $F_{x}$, and root mean square for component $F_{x}$

\begin{tabular}{|c|c|c|c|c|c|c|c|}
\hline $\begin{array}{l}\text { Network } \\
\text { No }\end{array}$ & $\begin{array}{l}\text { Network } \\
\text { Name }\end{array}$ & $\begin{array}{l}\text { Quality } \\
\text { (Training, \%) }\end{array}$ & $\begin{array}{l}\text { Quality } \\
\text { (Validation, \%) }\end{array}$ & $\begin{array}{l}\text { Error } \\
\text { (Training) }\end{array}$ & $\begin{array}{l}\text { Error } \\
\text { (Validation) }\end{array}$ & $\begin{array}{l}\text { Activation } \\
\text { (Hidden) }\end{array}$ & $\begin{array}{l}\text { Activation } \\
\text { (Output) }\end{array}$ \\
\hline \multicolumn{8}{|c|}{ Cutting force component $F_{x}$} \\
\hline 1 & MLP 2-2-1 & 97.35 & 99.98 & 1934.4 & 4222.3 & Tanh & Sinus \\
\hline \multicolumn{8}{|c|}{ Cutting force component $F_{y}$} \\
\hline 2 & MLP 2-6-1 & 99.95 & 99.74 & 10.1 & 467.8 & Tanh & Linear \\
\hline \multicolumn{8}{|c|}{ Amplitude of cutting force component $F_{x}\left(F_{x \_a m p}\right)$} \\
\hline 3 & MLP 2-9-1 & 99.12 & 99.99 & 1956.5 & 2365.9 & Logistic & Linear \\
\hline \multicolumn{8}{|c|}{ Root mean square of cutting force component $F_{x}\left(F_{x_{-} r m s}\right)$} \\
\hline 4 & RBF 2-9-1 & 99.73 & 99.95 & 74.2 & 263.8 & Gaussian & Linear \\
\hline
\end{tabular}

Table 4 presents the $\mathrm{R}^{2}$ correlation coefficients and sensitivity analysis of input parameters $\left(f_{z}\right.$ and $\left.v_{c}\right)$ of the analysed networks. It can be seen that neural networks are an appropriate tool to predict assumed values, and that both input parameters have a significant impact on these values. Moreover, the charts presented in Fig. 13 show this correlation (between experimental results and those obtained in the simulation). The charts present a correlation for networks which are presented in a visual form in Fig. 12 .

Based on the charts presented above, it can be stated that the networks obtained show satisfactory predictive capacity. All obtained correlation coefficients are higher than 0.95 . Thus, it can be stated that neural networks may be an effective tool for the simulation of, for example, cutting force components and their amplitudes or their root mean square. Neural networks may be effectively used for the numerical modelling of machining processes.

\section{Conclusions}

This paper presents the results of experimental study of the AZ31 magnesium alloy milling process. Two different tools were used in experimental studies, a PCD and a TiAlNcoated tool. First, modal analysis was performed to obtain a stability lobe diagram which was next verified for selected points by means of statistics and the multiscale entropy method. Additionally, a prediction was made of cutting force components and the amplitudes of components of cutting force $F_{a m p}$ and root mean square $F_{r m s}$ for the TiAlN-coated tool.

A novel and rarely employed approach was used in the analysis of the experimentally obtained signals. The SLD curves obtained using CutPro commercial software were verified for selected processing parameters. This is because these curves were obtained based on the static parameters of the machine-holder-workpiece-tool (MHWT) system.
During processing, these parameters may change as a result of the occurrence of dynamic phenomena, which affects the location of stability lobes. Then, the cutting force signals were subjected to a thorough analysis. Apart from the classic and commonly employed approach, a dynamic analysis was performed using multiscale entropy and numerical simulations using artificial neural networks. The obtained results confirm the appropriateness of the proposed research approach. The analysis using dynamic indices enables to analyse both the limit values of measured forces and the signal complexity, which significantly contributes to the quality of the performed studies.

Based on the studies conducted, it can be stated that:

1) Increasing the cutting speed at low ranges of values causes an increase in cutting force in all directions.

2) Performance of the machining process with high cutting speed values allows for component values of cutting force to be lowered as a result of the transition into the HSM range.

3) Increasing the feed per tooth value causes a linear increase in the values of all cutting force components, regardless of the technological parameters or type of cutting tool.

4) Regardless of the technological parameters or type of cutting tool, the highest values for the analysed parameters were recorded in most cases for the component $F_{x}$, whereas the lowest were recorded for $F_{y}$.

5) Lower values of parameters for milling with variable feed per tooth were obtained when using a tool with a TiAlN coating, something which was particularly noticeable for the component $F_{x}$. Nevertheless, in the case of changes in the cutting speed, the type of tool used did not show an unambiguous impact on the results obtained.

6) An analysis of entropy indicated a lower level of disorder for cutting force component signals recorded at 

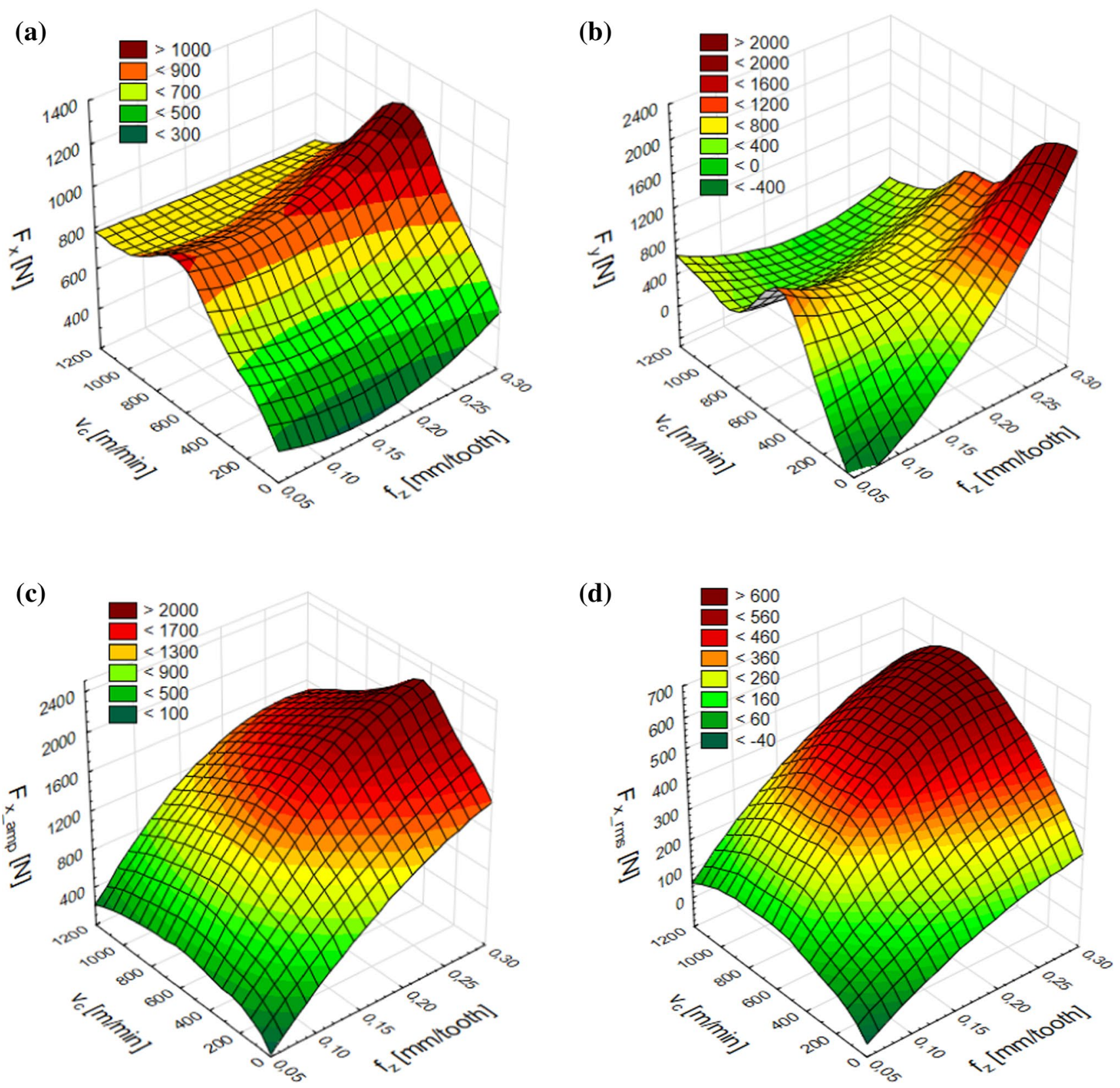

Fig. 12 Numerical results from network depending on the cutting speed $v_{c}$ and feed per tooth $f_{z}$ for a maximum cutting force component $F_{x}$, b maximum cutting force component $F_{y}, \mathbf{c}$ amplitude of cutting force component $F_{x}, \mathbf{d}$ root mean square of cutting force component $F_{x}$

Table 4 Correlation $R^{2}$ and sensitivity analysis of input parameters for the analysed networks

\begin{tabular}{lllll}
\hline & $F_{x}$ & $F_{y}$ & $F_{x_{\_} a m p}$ & $F_{x_{-} r m s}$ \\
\hline Network & MLP 2-2-1 & MLP 2-6-1 & MLP 2-9-1 & RBF 2-9-1 \\
Corelation $R^{2}$ & 0.9658 & 0.9986 & 0.9896 & 0.9952 \\
Sensitivity analysis $f_{z} / v_{c}$ & $35.03 / 11.01$ & $1392.78 / 269.03$ & $2871.62 / 39.00$ & $84.87 / 62.56$ \\
\hline
\end{tabular}


(a)

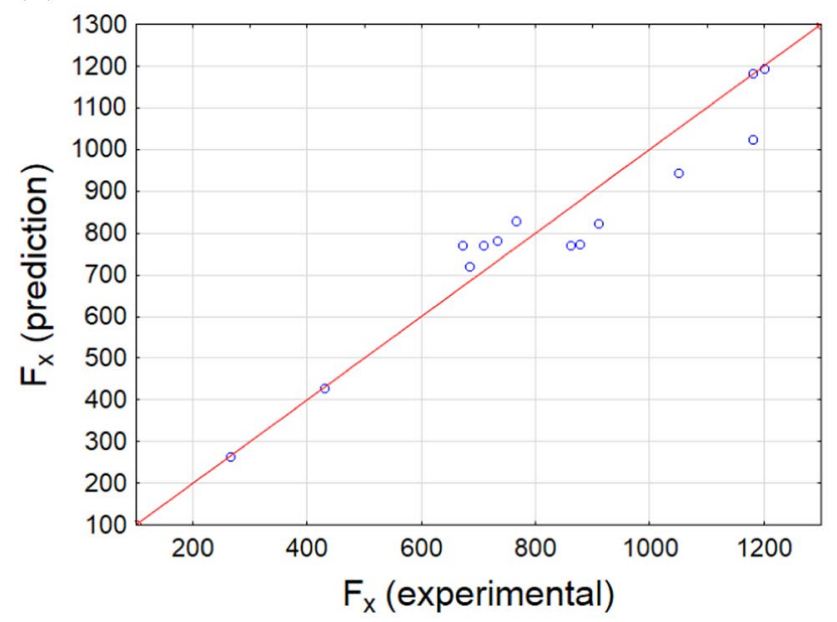

(c)

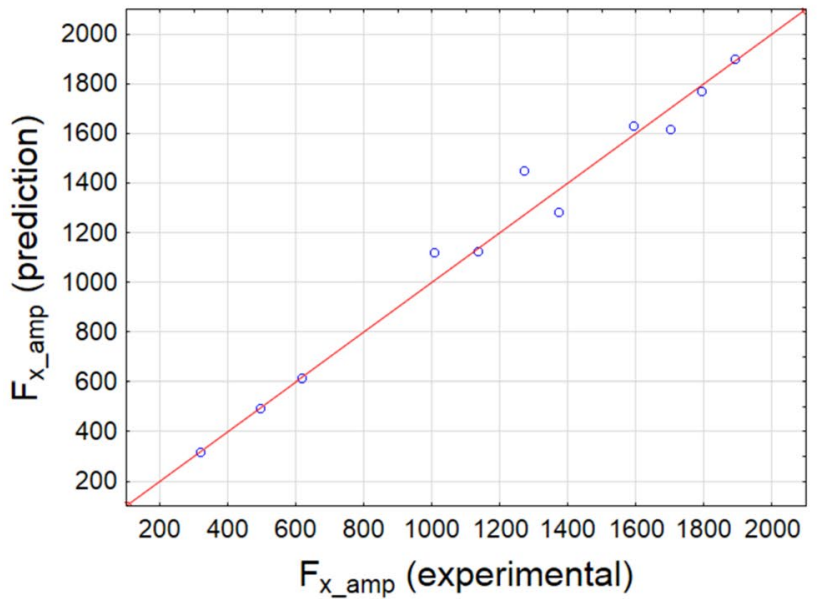

(b)

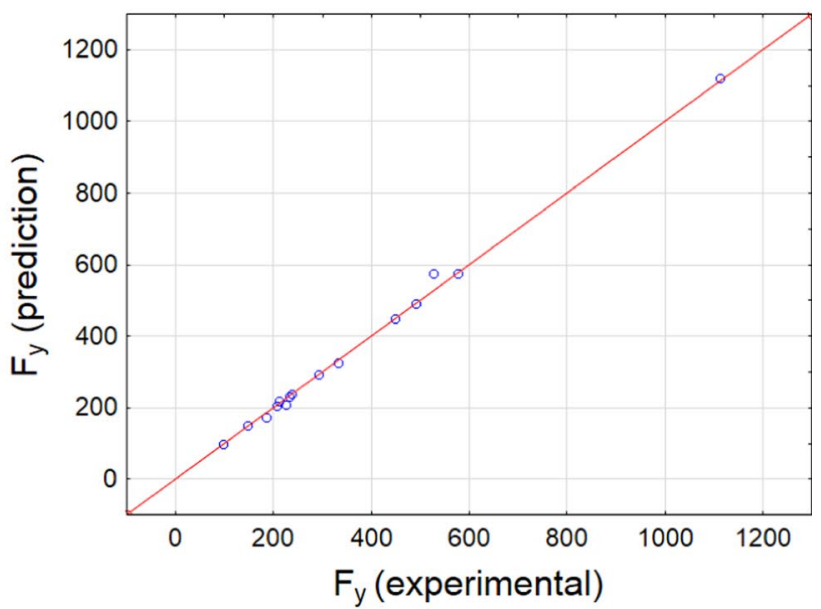

(d)

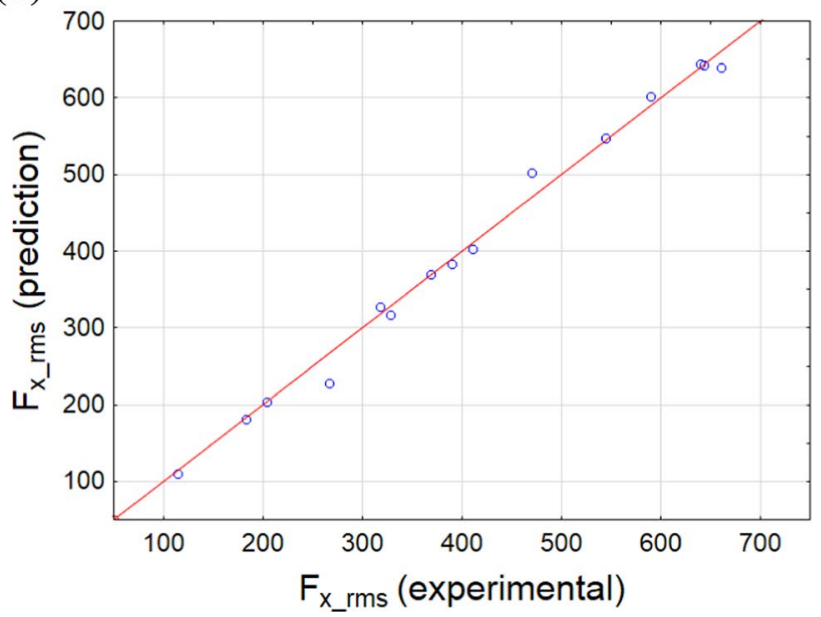

Fig. 13 Comparison of experimental and numerical results a cutting force component $F_{x}$, b cutting force component $F_{y}$, $\mathbf{c}$ amplitude of cutting force component $F_{x}$, d Root mean square of cutting force component $F_{x}$

the highest cutting speed of $v_{c}=1200 \mathrm{~m} / \mathrm{min}$ and at the highest feed per tooth of $f_{z}=0.30 \mathrm{~mm} /$ tooth.

7) During milling with variable cutting speeds, a lower level of disorder was observed for cutting force component signals recorded when milling using the PCD tool, while when milling with changes in the feed per tooth this lower level was observed for the TiAlN-coated tool.

8) It is possible to predict the impact of technological parameters $\left(f_{z}\right.$ and $\left.v_{c}\right)$ on the values of cutting force components as well as on the value of the amplitudes of cutting forces $F_{a m p}$ and root mean square $F_{r m s}$. In most cases, better results were obtained for the MLP neural network than for RBF.

9) The modelling of neural networks may be an effective tool for predicting the components of total cutting force as well as for the amplitudes of components of cutting force $F_{\text {amp }}$ and root mean square $F_{r m s}$. The $R^{2}$ correlation coefficients were respectively $F_{x}-0.9658, F_{y}-0.9986$, $F_{x_{\_} a m p}-0.9896$, and $F_{x_{-} r m s}-0.9952$; thus it can be stated that the trained networks are a reliable predictor of utility values of cutting indicators.

10) The results of modelling and the simulations conducted may be used to create tools while establishing machining conditions in industrial settings, as support for technicians in the design of technological processes. Excessively high cutting force values may have a negative impact on the deformation of machined elements.

Author contributions Conceptualization: IZ, AW, MK; Methodology: IZ, AW, JK, MK; Formal analysis and investigation: IZ, AW, JK, MK; 
Writing—original draft preparation: IZ, AW, JK, MK; Writing—review and editing: IZ, MK, RR; Funding acquisition: MK; Resources: IZ, MK; Supervision: IZ, MK.

Funding The project/research was financed in the framework of the project Lublin University of Technology-Regional Excellence Initiative, funded by the Polish Ministry of Science and Higher Education (contract no. 030/RID/2018/19).

Data availability The raw/processed data required to reproduce these findings cannot be shared at this time as the data also forms part of an ongoing study.

\section{Code availability Sieć MLP 2-2-1}

$<$ ? xml version $=$ "1.0" "encoding $=$ "UTF-8"? $>$

$<$ PMML version = "3.0" $><$ Header copyright $=$ "Copyright 1984-2017 TIBCO Software Inc. All rights reserved." > < Application name $=$ "STATISTICA Automated Neural Networks $($ SANN)" version $=" 2.0 " /></$ Header $><$ DataDictionary numberOfFields $=" 3 "><$ DataField name $=$ "Fx" optype $=$ "continuous" $/><$ DataField name $=$ "fz" optype $=$ "continuous" $/><$ DataField name $=$ "vc" optype $=$ "continuous" $/></$ DataDictionary $><$ NeuralNetwork modelName $=$ "A $1-\mathrm{k}_{-}$ MLP 2-2-1" functionName = "regression" $><$ MiningSchema $><$ MiningField name $=" F x "$ usageType $=$ "predicted"/ > MiningField name $=$ "fz" low Value $=" 0.050000 "$ highValue $=" 0.300000 " /><$ MiningField name $=" v c "$ low Value $=" 200.000000 "$ highValue $=" 1200.000000 " /></$ MiningSchema $><$ NeuralInputs numberOfInputs $=" 2 "><\mathrm{Neu}-$ ralInput id $=" 0 "><$ DerivedField $><$ NormContinuous field $=" f z "$ shift $="-2.00000000000000 \mathrm{e}-001 "$ scale $=" 4.00000000000000 \mathrm{e}+000 "><$ LinearNorm orig $=" 5.00000000000000 \mathrm{e}-002 "$ norm $=" 0.000000 " /><$ LinearNorm orig $=" 3.00000000000000 \mathrm{e}-001 "$ norm $=" 1.000000 " /></$ NormContinuous $></$ DerivedField $><$ /NeuralInput $><$ NeuralInput id $=" 1 "><$ DerivedField $><$ NormContinuous field $=$ "vc" shift $="-2.00000000000000 \mathrm{e}-001 "$ scale $=" 1.00000000000000 \mathrm{e}-$ $003 "><$ LinearNorm orig $=" 2.00000000000000 \mathrm{e}+002 "$ norm $=" 0.000000 " /><$ LinearNorm orig $=" 1.20000000000000$ $\mathrm{e}+003 "$ norm $=" 1.000000 " /></$ NormContinuous $></$ DerivedField $><$ /NeuralInput $><$ /NeuralInputs $><$ NeuralLayer numberOfNeurons $=" 2 "$ activationFunction $=" \tanh "><$ Neuron id $=" 2 "$ bias $="-1.18589924841596 \mathrm{e}+000 "><$ Con from $=$ "0" weight $=$ $"-3.11229883898561 \mathrm{e}+000 " /><$ Con from $=" 1$ " weight $=" 9.990$ $02570918014 \mathrm{e}+000 " /></$ Neuron $><$ Neuron id $=" 3 "$ bias $=" 4$. $86682950265097 \mathrm{e}+000 "><$ Con from $=" 0$ " weight $="-5.420052$ $87404041 \mathrm{e}+000 " /><$ Con from $=" 1 "$ weight $="-1.12476163604$ $482 \mathrm{e}+001 " /></$ Neuron $></$ NeuralLayer $><$ NeuralLayer numberOfNeurons $=" 1$ " activationFunction $=$ "sine" $><$ Neuron id $=" 4 "$ bias $=" 4.14031536478860 \mathrm{e}-001 "><$ Con from $=" 2$ " weight $="-1.251$ $64661820436 \mathrm{e}+001 " /><$ Con from $=" 3$ " weight $="-1.267551283688$ $54 \mathrm{e}+001 " /></$ Neuron $></$ NeuralLayer $><$ NeuralOutputs numberOfOutputs $=" 1 "><$ NeuralOutput outputNeuron $=" 4 "><$ DerivedField optype $=$ "continuous" $><$ NormContinuous field $=" F x "$ shift $="-2.82051282051282 \mathrm{e}-001 "$ scale $=" 1.06837606837607 \mathrm{e}-$ $003 "><$ LinearNorm orig $=" 2.64000000000000 \mathrm{e}+002 "$ norm $=" 0.00000000000000 \mathrm{e}+000 " /><$ LinearNorm orig $=" 1$. $20000000000000 \mathrm{e}+003 "$ norm $=" 1.00000000000000 \mathrm{e}+000 " /$ $><$ /NormContinuous $></$ DerivedField $><$ /NeuralOutput $></$ NeuralOutputs $></$ NeuralNetwork $></$ PMML $>$

Sieć MLP 2-6-1

$<$ ?xml version $=" 1.0 "$ encoding $=" \mathrm{UTF}-8 "$ ? $>$

$<$ PMML version $=" 3.0 "><$ Header copyright $=$ "Copyright 1984-2017 TIBCO Software Inc. All rights reserved." > < Application name $=$ "STATISTICA Automated Neural Networks
$($ SANN $) "$ version $=" 2.0 " /></$ Header $><$ DataDictionary numberOfFields $=" 3 "><$ DataField name $=$ "Fy" optype $=$ "continuous" $/><$ DataField name $=$ "fz" optype $=$ "continuous" $/><$ DataField name $=$ "vc" optype $=$ "continuous" $/></$ DataDictionary $><$ NeuralNetwork modelName $=$ "Dane dl_MLP 2-6-1" functionName $=$ "regression" $><$ MiningSchema $><$ MiningField name $=$ "Fy" usageType $=$ "predicted"/ > MiningField name $=$ "fz" lowValue = "0.050000" highValue $=" 0.300000 " /><$ MiningField name $=" v c "$ lowValue $=" 200.000000 "$ highValue $=" 1200.000000 " /></$ MiningSchema $><$ NeuralInputs numberOfInputs $=" 2 "><\mathrm{Neu}-$ ralInput id $=" 0 "><$ DerivedField $><$ NormContinuous field $=$ "fz" shift $="-2.00000000000000 \mathrm{e}-001 "$ $\mathrm{scale}=" 4.00000000000000 \mathrm{e}+000 "><$ LinearNorm orig $=" 5.00000000000000 \mathrm{e}-002 "$ norm $=" 0.000000 " /><$ Linear Norm orig $=" 3.00000000000000 \mathrm{e}-001 "$ norm $=" 1.000000 " /></$ NormContinuous $><$ /DerivedField $><$ /NeuralInput $><$ NeuralInput id $=" 1 "><$ DerivedField $><$ NormContinuous field $=$ "vc" shift $="-2.00000000000000 \mathrm{e}-001 "$ scale $=" 1.00000000000000 \mathrm{e}-$ $003 "><$ LinearNorm orig $=" 2.00000000000000 \mathrm{e}+002 "$ norm $=" 0.000000 " /><$ LinearNorm orig $=" 1.20000000000000$ $\mathrm{e}+003 "$ norm $=" 1.000000 " /></$ NormContinuous $></$ DerivedField $><$ /NeuralInput $><$ /NeuralInputs $><$ NeuralLayer numberOfNeurons $=" 6 "$ activationFunction $=$ "tanh" $><$ Neuron id $=" 2 "$ bias $=" 9.16077678635015 \mathrm{e}+000 "><$ Con from $=" 0 "$ weight $=" 6.01837602778105 \mathrm{e}-001 " /><$ Con from $=" 1 "$ wei ght $=" 5.82694078133876 \mathrm{e}+000 " /></$ Neuron $><$ Neuron id = "3" bias $="-1.01907749095250 \mathrm{e}+000 "><$ Con from $=" 0 "$ weight $="-4.95428801320388 \mathrm{e}-001 " /><$ Con from $=" 1$ " weight $=$ "4.83869212777818e + 000"/ > </Neuron $><$ Neuron id = "4" bia $\mathrm{s}=" 6.32992536199350 \mathrm{e}+000 "><$ Con from $=" 0 "$ weight $=" 2.3$ $5693811320159 \mathrm{e}+001 " /><$ Con from $=$ "1" weight $="-5.19456$ $918989006 \mathrm{e}+001 " /></$ Neuron $><$ Neuron id $=" 5 "$ bias $="-5.2$ $2706775808340 \mathrm{e}+000 "><$ Con from $=$ "0" weight $=" 7.6277619$ $7170835 \mathrm{e}+000 " /><$ Con from $=" 1 "$ weight $=" 3.685784851887$ $40 \mathrm{e}+001 " /></$ Neuron $><$ Neuron id $=" 6 "$ bias $=" 2.421072240$ $87247 \mathrm{e}+001 "><$ Con from $=" 0 "$ weight $=" 2.72924735009545 \mathrm{e}$ $+001 " /><$ Con from $=" 1 "$ weight $="-3.31596231243579 \mathrm{e}+001$ $" /></$ Neuron $><$ Neuron id $=" 7 "$ bias $="-2.98010600504767 \mathrm{e}-$ $001 "><$ Con from $=" 0 "$ weight $="-6.14657209571972 \mathrm{e}-$ $002 " /><$ Con from $=" 1 "$ weight $=" 1.01557638112404 \mathrm{e}-002 " /></$ Neuron $></$ NeuralLayer $><$ NeuralLayer numberOfNeurons $=" 1 "$ activationFunction $=$ "identity" $><$ Neuron $\mathrm{id}=" 8$ " bias $=" 6.8759$ $3873105184 \mathrm{e}+000 "><$ Con from $=" 2$ " weight $="-5.962291148$ $52509 \mathrm{e}+000 " /><$ Con from $=$ "3" weight $="-5.1166562107657$ $4 \mathrm{e}+000 " /><$ Con from $=" 4 "$ weight $="-3.17158638187285 \mathrm{e}+0$ $00 " /><$ Con from $=" 5 "$ weight $=" 1.52596438545984 \mathrm{e}+000 " />$ $<$ Con from $=" 6 "$ weight $="-5.42228562354627 \mathrm{e}+000 " /><$ Con from $=" 7$ " weight $="-1.65370312406208 \mathrm{e}+001 " /></$ Neuron $></$ NeuralLayer $><$ NeuralOutputs numberOfOutputs $=" 1 "><$ NeuralOutput outputNeuron $=" 8 "><$ DerivedField optype $=$ "continuous" $><$ NormContinuous field $=$ "Fy" shift $="-2.04592901878914 \mathrm{e}-$ $001 "$ scale $=" 2.08768267223382 \mathrm{e}-003 "><$ LinearNorm orig $=" 9.80$ $000000000000 \mathrm{e}+001 "$ norm $=" 0.00000000000000 \mathrm{e}+000 " /><$ Lin earNorm orig $=" 5.77000000000000 \mathrm{e}+002 "$ norm $=" 1.0000000$ $0000000 \mathrm{e}+000 " /></$ NormContinuous $></$ DerivedField $><1$ NeuralOutput $><$ /NeuralOutputs $><$ /NeuralNetwork $></$ PMML $>$

Sieć MLP 2-9-1

$<$ ? xml version $=" 1.0 "$ encoding $=" \mathrm{UTF}-8 "$ ? $>$

$<$ PMML version = "3.0" > < Header copyright $=$ "Copyright 1984-2017 TIBCO Software Inc. All rights reserved." > < Application name $=$ "STATISTICA Automated Neural Networks $($ SANN $) "$ version $=" 2.0 " /></$ Header $><$ DataDictionary numberOfFields $=" 3 "><$ DataField name $=$ "Fx_amp" optype $=$ "continuous" $/><$ DataField name $=$ "fz" optype $=$ "continuous" $/><$ DataField name $=$ "vc" optype $=$ "continuous" $/></$ 
DataDictionary $><$ NeuralNetwork modelName $=$ "Arku MLP 2-9-1" functionName = "regression" $><$ MiningSchema $><$ MiningField name $=$ "Fx_amp" usageType $=$ "predicted"/ $><$ MiningField name $=$ "fz" lowValue $=" 0.050000 "$ highValue $=" 0.300000 " /><$ MiningField name $=$ vc" lowValue $=" 200.000000 "$ highValue $=" 1000.000000 " /></$ MiningSchema $><$ NeuralInputs numberOfInputs $=" 2 "><\mathrm{Neu}-$ rallnput id $=" 0 "><$ DerivedField $><$ NormContinuous field $=" f z "$ shift $="-2.00000000000000 \mathrm{e}-001 "$ scale $=" 4.00000000000000 \mathrm{e}+000 "><$ LinearNorm orig $=" 5.00000000000000 \mathrm{e}-002 "$ norm $=" 0.000000 " /><$ LinearNorm orig $=" 3.00000000000000 \mathrm{e}-001 "$ norm $=" 1.000000 " /></$ NormContinuous $></$ DerivedField $></$ NeuralInput $><$ NeuralInput id $=" 1 "><$ DerivedField $><$ NormContinuous field $=$ "vc" shift $="-2.50000000000000 \mathrm{e}-001 "$ scale $=" 1.25000000000000 \mathrm{e}-$ $003 "><$ LinearNorm orig $=" 2.00000000000000 \mathrm{e}+002 "$ norm $=" 0.000000 " /><$ LinearNorm orig $=" 1.00000000000000 \mathrm{e}+0$ 03" norm $=" 1.000000 " /></$ NormContinuous $></$ DerivedField $></$ NeuralInput $></$ NeuralInputs $><$ NeuralLayer numberOfNeurons $=$ "9" activationFunction $=$ "logistic" $><$ Neuron id $=" 2 "$ bias $=$ $" 1.33737103868989 \mathrm{e}+001 "><$ Con from $=$ "0" weight $=" 1.965703$ $19259705 \mathrm{e}+001 " /><$ Con from $=" 1 "$ weight $=" 1.80936204857776$ $\mathrm{e}+001 " /></$ Neuron $><$ Neuron id $=" 3$ " bias $=" 6.4610112910942$ $1 \mathrm{e}+000 "><$ Con from $=$ "0" weight $=" 1.89621342542113 \mathrm{e}+001 " /$ $><$ Con from $=" 1$ " weight $=" 1.62657345172437 \mathrm{e}+001 " /></ \mathrm{Neu}-$ ron $><$ Neuron id $=" 4 "$ bias $="-1.99602077190091 \mathrm{e}+001 "><$ Con from $=$ " 0 " weight $=" 2.77014743102729 \mathrm{e}+001 " /><$ Con from $=" 1 "$ weight $=" 4.72963466548396 \mathrm{e}+001 " /></$ Neuron $><$ Neuron $\mathrm{id}=" 5$ " bias $=" 4.76560004902435 \mathrm{e}+000 "><$ Con from $=" 0 "$ wei ght $=" 2.44890603958559 \mathrm{e}+001 " /><$ Con from $=" 1$ " weight $=" 1.4$ $6195383161955 \mathrm{e}+001 " /></$ Neuron $><$ Neuron id ="6"bias $=" 2$. $70085898407752 \mathrm{e}+000 "><$ Con from $=$ " 0 " weight $=$ " 6.46027926 $189907 \mathrm{e}+000 " /><$ Con from $=" 1 "$ weight $=" 1.53994873279955 \mathrm{e}-$ $002 " /></$ Neuron $><$ Neuron id $=" 7 "$ bias $=" 1.34639913110717 \mathrm{e}$ $+001 "><$ Con from $=$ "0" weight $=" 1.82579596322517 \mathrm{e}+001 " />$ $<$ Con from $=" 1 "$ weight $=" 1.60364007176658 \mathrm{e}+001 " /></$ Neuron $><$ Neuron id $=" 8 "$ bias $=" 4.07950902087682 \mathrm{e}-001 "><$ Con from $=$ "0" weight $="-6.28181847515253 \mathrm{e}-002 " /><$ Con from $=" 1 "$ weight $=" 6.68650024916436 \mathrm{e}-002 " /></$ Neuron $><$ Neuron id $=" 9 "$ bias $=" 1.11333524858615 \mathrm{e}+001 "><$ Con from $=" 0 "$ weight $="-2$. $74954018958666 \mathrm{e}+001 " /><$ Con from $=" 1$ " weight $=" 5.7321710$ $1377037 \mathrm{e}+001 " /></$ Neuron $><$ Neuron id $=" 10 "$ bias $=" 3.6132$ $2473112573 \mathrm{e}+000 "><$ Con from $=$ "0" weight $=" 1.69297869827$ $781 \mathrm{e}+001 " /><$ Con from $=" 1 "$ weight $=" 1.41479875306435 \mathrm{e}+0$ $01 " /></$ Neuron $></$ NeuralLayer $><$ NeuralLayer numberOfNeurons $=" 1$ " activationFunction $=$ "identity" $><$ Neuron id $=" 11$ " bia $\mathrm{s}=" 3.12526611515799 \mathrm{e}+000 "><$ Con from $=" 2$ " weight $=" 6.42$ $106021497384 \mathrm{e}+000 " /><$ Con from $=" 3$ " weight $=" 4.911183628$ $87815 \mathrm{e}+000 " /><$ Con from $=" 4 "$ weight $=" 1.48545472652851 \mathrm{e}$ $+001 " /><$ Con from $=$ "5" weight $="-6.61578771744833 \mathrm{e}+000 " /$ $><$ Con from $=$ "6" weight $=" 9.81719562718128 \mathrm{e}+000 " /><$ Con from $=$ "7" weight $=" 1.07684841114401 \mathrm{e}+001 " /><$ Con from $=" 8 "$ weight $="-2.64076240956808 \mathrm{e}+001 " /><$ Con from $=$ "9" weight $="-2.98683784829308 \mathrm{e}+001 " /><$ Con from $=" 10 "$ weight $=" 3$. $37435868452225 \mathrm{e}+000 " /></$ Neuron $></$ NeuralLayer $><$ NeuralOutputs numberOfOutputs $=" 1 "><$ NeuralOutput outputNeuron $=" 11 "><$ DerivedField optype $=$ "continuous" $><$ NormContinuous field $=$ "Fx_amp" shift $=$ "-2.02290076335878e-001" scale $=" 6.36132315521629 \mathrm{e}-004 "><$ LinearNorm orig $=" 3.1800$ $0000000000 \mathrm{e}+002 "$ norm $=" 0.00000000000000 \mathrm{e}+000 " /><$ LinearNorm orig $=" 1.89000000000000 \mathrm{e}+003 "$ norm $=" 1.0000000$ $0000000 \mathrm{e}+000 " /></$ NormContinuous $></$ DerivedField $></$ NeuralOutput $><$ /NeuralOutputs $></$ NeuralNetwork $></$ PMML $>$

Sieć RBF 2-9-1

$<$ ? $\mathrm{xml}$ version $=" 1.0 "$ encoding $=$ "UTF-8"? >
$<$ PMML version $=" 3.0 "><$ Header copyright $=$ "Copyright 1984-2017 TIBCO Software Inc. All rights reserved." > < Application name $=$ "STATISTICA Automated Neural Networks $($ SANN $) "$ version $=" 2.0 " /></$ Header $><$ DataDictionary numberOfFields $=" 3 "><$ DataField name $=$ "Frms_x" optype $=$ "continuous" $/><$ DataField name $=$ "fz" optype = "continuous" $/><$ DataField name $=$ "vc" optype $=$ "continuous" $/></$ DataDictionary $><$ NeuralNetwork modelName $=" \mathrm{~A} 1-\mathrm{k}_{-}$ RBF 2-9-1" functionName = "regression" $><$ Mining Schema $><$ MiningField name $=$ "Frms_x" usageType $=$ "predicted" $/><$ MiningField name $="$ fz" lowValue $=" 0.050000 "$ highValue $=" 0.300000 " /><$ MiningField name $=" v c "$ lowValue $=" 200.000000 "$ highValue $=" 1200.000000 " /></$ MiningSchema $><$ NeuralInputs numberOfInputs $=" 2 "><\mathrm{Neu}-$ ralinput id $=" 0 "><$ DerivedField $><$ NormContinuous field $=$ "fz" shift $="-2.00000000000000 \mathrm{e}-001 "$ scale $=44.00000000000000 \mathrm{e}+000 "><$ LinearNorm orig $=" 5.00000000000000 \mathrm{e}-002 "$ norm $=" 0.000000 " /><$ Linear Norm orig $=" 3.00000000000000 \mathrm{e}-001 "$ norm $=" 1.000000 " /></$ NormContinuous $><$ /DerivedField $><$ /NeuralInput $><$ NeuralInput id $=" 1 "><$ DerivedField $><$ NormContinuous field $=$ "vc" shift $="-2.00000000000000 \mathrm{e}-001 "$ scale $=" 1.00000000000000 \mathrm{e}-$ $003 "><$ LinearNorm orig = "2.00000000000000e + 002" norm $=" 0.000000 " /><$ LinearNorm orig $=" 1.20000000000000$ $\mathrm{e}+003 "$ norm $=" 1.000000 " /></$ NormContinuous $></$ DerivedField $></$ NeuralInput $></$ NeuralInputs $><$ NeuralLayer numberOfNeurons $=" 9 "$ activationFunction $=$ "radialBasis" $><$ Neuron $\mathrm{id}=$ "2" width $=" 1.00000000000000 \mathrm{e}-001 "><$ Con from $=" 0 "$ weight $=" 4.00000000000000 \mathrm{e}-001 " /><$ Con from $=" 1 "$ weight $=" 9.00000000000000 \mathrm{e}-001 " /></$ Neuron $><$ Neuron $\mathrm{id}=$ "3" width $=$ "9.99999999999999e-002" > < Con from $=" 0 "$ weight $=" 4.00000000000000 \mathrm{e}-001 " /><$ Con from $=" 1 "$ weight $=" 7.00000000000000 \mathrm{e}-001 " /></$ Neuron $><$ Neuron $\mathrm{id}=" 4 "$ width $=$ "9.99999999999999e-002" > < Con from $=" 0 "$ weight $=" 4.00000000000000 \mathrm{e}-001 " /><$ Con from $=" 1 "$ weight $=" 6.00000000000000 \mathrm{e}-001 " /></$ Neuron $><$ Neuron $\mathrm{id}=" 5 "$ width $=" 2.00000000000000 \mathrm{e}-001 "><$ Con from $=" 0 "$ weight $=" 4.00000000000000 \mathrm{e}-001 " /><$ Con from $=" 1 "$ wei ght $=" 0.00000000000000 \mathrm{e}+000 " /></$ Neuron $><$ Neuron id $=" 6 "$ width $=" 1.00000000000000 \mathrm{e}-001 "><$ Con from $=" 0 "$ weight $=" 4.00000000000000 \mathrm{e}-001 " /><$ Con from $=" 1 "$ weight $=" 2.00000000000000 \mathrm{e}-001 " /></$ Neuron $><$ Neuron $\mathrm{id}=" 7 "$ width $=" 1.00000000000000 \mathrm{e}-001 "><$ Con from $=" 0 "$ weight $=" 4.00000000000000 \mathrm{e}-001 " /><$ Con from $=" 1 "$ weight $=" 3.00000000000000 \mathrm{e}-001 " /></$ Neuron $><$ Neuron $\mathrm{id}=" 8 "$ width $=" 1.00000000000000 \mathrm{e}-001 "><$ Con from $=" 0 "$ weight $=" 4.00000000000000 \mathrm{e}-001 " /><$ Con from $=" 1 "$ weight $=" 8.00000000000000 \mathrm{e}-001 " /></$ Neuron $><$ Neuron id $=" 9 "$ width $=" 1.00000000000000 \mathrm{e}-001 "><$ Con from $=" 0 "$ weight $=" 4.00000000000000 \mathrm{e}-001 " /><$ Con from $=" 1 "$ weight $=" 5.00000000000000 \mathrm{e}-001 " /></$ Neuron $><$ Neuron $\mathrm{id}=" 10 "$ width $=" 6.00000000000000 \mathrm{e}-001 "><$ Con from $=" 0 "$ weight $=" 1.00000000000000 \mathrm{e}+000 " /><$ Con from $=" 1 "$ weight $=" 6.00000000000000 \mathrm{e}-001 " /></$ Neuron $></$ NeuralLayer $><$ NeuralLayer numberOfNeurons $=" 1 "$ activationFunction $=$ "identity" $><$ Neuron id $=$ "11" bias $=$ "-3.26915420082916e$001 "><$ Con from $=" 2$ " weight $=" 2.20601468486232 \mathrm{e}-001 " /><$ Con from $=" 3$ " weight $=" 9.40700728115262 \mathrm{e}-001 " /><$ Con from $=" 4 "$ weight $="-5.21245667594871 \mathrm{e}-001 " /><$ Con from $=" 5 "$ weight $=" 1.31646831970374 \mathrm{e}-001 " /><$ Con from $=" 6 "$ weight $="-9.39266928160472 \mathrm{e}-001 " /><$ Con from $=" 7 "$ weight $=" 1.12820217709549 \mathrm{e}+000 " /><$ Con from $=" 8 "$ weight $="-5.17628419352205 \mathrm{e}-001 " /><$ Con from $=" 9 "$ weight $=" 7.08016955495058 \mathrm{e}-002 " /><$ Con from $=" 10 "$ wei ght $=" 1.28932490272739 \mathrm{e}+000 " /></$ Neuron $></$ NeuralLayer $><$ NeuralOutputs numberOfOutputs $=" 1 "><$ NeuralOutput 
outputNeuron $=" 11 "><$ DerivedField optype $=$ "continuous" $><$ NormContinuous field $=$ "Frms_x" shift $="-2.08791208791209 \mathrm{e}-001 "$ scale $=" 1.83150183150183 \mathrm{e}-003 "><$ LinearNorm orig $=" 1.1400$ $0000000000 \mathrm{e}+002 "$ norm $=" 0.00000000000000 \mathrm{e}+000 " /><$ LinearNorm orig $=" 6.60000000000000 \mathrm{e}+002 "$ norm $=" 1.0000000$ $0000000 \mathrm{e}+000 " /></$ NormContinuous $></$ DerivedField $></$ NeuralOutput $></$ NeuralOutputs $></$ NeuralNetwork $></$ PMML $>$

\section{Declarations}

Conflict of interest The author declares that he has no conflict of interest.

Open Access This article is licensed under a Creative Commons Attribution 4.0 International License, which permits use, sharing, adaptation, distribution and reproduction in any medium or format, as long as you give appropriate credit to the original author(s) and the source, provide a link to the Creative Commons licence, and indicate if changes were made. The images or other third party material in this article are included in the article's Creative Commons licence, unless indicated otherwise in a credit line to the material. If material is not included in the article's Creative Commons licence and your intended use is not permitted by statutory regulation or exceeds the permitted use, you will need to obtain permission directly from the copyright holder. To view a copy of this licence, visit http://creativecommons.org/licenses/by/4.0/.

\section{References}

1. Cus F, Zuperl U, Gecevska V. High-speed milling of light metals. J Achiev Mater Manuf Eng. 2007;24(1):357-64.

2. Zawada-Michalowska M, Jozwik J, Legutko S, Mika D, Pieśko P, Pytka J. Cutting force during surface layer milling of selected aluminium alloys. Materials. 2020;13:5725. https://doi.org/10. 3390/ma13245725.

3. Weremczuk A, Kecik K, Rusinek R, Warminski J. The dynamics of the cutting process with duffing nonlinearity. Maint Reliability. 2013;15:209-13.

4. Danis I, Monies F, Lagarrigue P, Wojtowicz N. Cutting forces and their modelling in plunge milling of magnesium-rare earth alloys. Int J Adv Manuf Technol. 2016;84(9-12):1801-20. https://doi. org/10.1007/s00170-015-7826-3.

5. Zgórniak P, Stachurski W, Ostrowski D. Application of thermographic measurements for the determination of the impact of selected cutting parameters on the temperature in the workpiece during milling process. J Mech Eng. 2016;62(11):657-64. https:// doi.org/10.5545/sv-jme.2015.3259.

6. Zagórski I, Kuczmaszewski J. Temperature measurements in the cutting zone, mass, chip fragmentation and analysis of chip metallography images during AZ31 and AZ91HP magnesium alloy milling. Aircr Eng Aerosp Technol. 2018;90(3):496-505. https:// doi.org/10.1108/AEAT-12-2015-0254.

7. Kuczmaszewski J, Zagórski I. Methodological problems of temperature measurement in the cutting area during milling magnesium alloys. Manag Prod Eng Rev. 2013;4(3):26-33. https://doi. org/10.2478/mper-2013-0025.

8. Adamski W. Manufacturing development strategies in aviation industry. Adv Manuf Sci Technol. 2010;34(3):73-84.
9. Oczoś KE, Kawalec A. Kształtowanie stopów lekkich. Wyd. Naukowe PWN; Warsaw; 2012.

10. Zagórski I, Kulisz M. The influence of technological parameters on cutting force components in milling of magnesium alloys with PCD tools and prediction with artificial neural networks. In: Gapiński B, Szostak M, Ivanov V, editors. Advances in manufacturing II. Cham: Springer; 2019. (MANUFACTURING 2019. Lecture Notes in Mechanical Engineering).

11. Shi K, Zhang D, Ren J, Yao Ch, Huang X. Effect of cutting parameters on machinability characteristics in milling of magnesium alloy with carbide tool. Adv Mech Eng. 2016;8(1):1-9. https:// doi.org/10.1177/1687814016628392.

12. Sivam SPSS, Bhat MDJ, Natarajan S, Chauhan N. Analysis of residual stresses, thermal stresses, cutting forces and other output responses of face milling operation on ZE41 Magnesium alloy. Int J Mod Manuf Technol. 2018;10(1):92-101.

13. Fu ZT, Yang WY, Wang XL, Leopold J. Analytical Modelling of Milling Forces for Helical End Milling Based on a Predictive Machining Theory. 15th CIRP Conference on Modelling of Machining Operations 2015;31:258-263.

14. Salguero J, Batista M, Calamaz M, Girot F, Marcos M. Cutting forces parametric model for the dry high speed contour milling of aerospace aluminium alloys. Procedia Eng. 2013;63:735-42. https://doi.org/10.1016/j.proeng.2013.08.215.

15. Kecik K, Borowiec M, Rusinek R. Verification of the stability lobes of Inconel 718 milling by recurrence plot applications and composite multiscale entropy analysis. Eur Phys J Plus. 2016;131:14. https://doi.org/10.1140/epjp/i2016-16014-x.

16. Weremczuk A, Rusinek R, Warminski J. The concept of active elimination of vibrations in milling process. Procedia CIRP. 2015;31:82-7. https://doi.org/10.1016/j.procir.2015.03.036.

17. Altintas Y, Stepan G, Merdol D, Dombovari Z. Chatter stability of milling in frequency and discrete time domain. CIRP J Manuf Sci Technol. 2008;1:35-44. https://doi.org/10.1016/j.cirpj.2008. 06.003.

18. Litak G, Syta A, Rusinek R. Dynamical changes during composite milling: recurrence and multiscale entropy analysis. Int $\mathrm{J}$ Adv Manuf Technol. 2011;56:445-53. https://doi.org/10.1007/ s00170-011-3195-8.

19. Bobrenkov OA, Khasawneh FA, Butcher EA, Mann BP. Analysis of milling dynamics for simultaneously engaged cutting teeth. J Sound Vib. 2010;329:585-606. https://doi.org/10.1016/j.jsv.2009. 09.032 .

20. Zagórski I, Kulisz M, Semeniuk A, Malec A. Artificial neural network modelling of vibration in the milling of AZ91D alloy. Adv Sci Technol Res J. 2017;11(3):261-9.

21. Weremczuk A, Rusinek R, Warminski J. Bifurcation and stability analysis of a nonlinear milling process. AIP Conference Proceedings 1922;100008. https://doi.org/10.1063/1.5019093.

22. Lipski J, Zaleski K. Optimisation of milling parameters using neural network. ITM Web Conference, 2017;15:01005. https:// doi.org/10.1051/itmconf/20171501005.

23. Kilickap E, Yardimeden A, Celik YH. Mathematical modelling and optimization of cutting force, tool wear and surface roughness by using artificial neural network and response surface methodology in milling of Ti-6242S. Appl Sci. 2017;7(10):1064. https:// doi.org/10.3390/app7101064.

24. Kazemi P, Khalid MH, Szlek J, Mirtic A, Reynolds G, Jachowicz R, Mendyk A. Computational intelligence modeling of granule size distribution for oscillating milling. Powder Technol. 2016;301:1252-8. 
25. Kulisz M, Zagórski I, Semeniuk A. Artificial neural network modelling of cutting force components during AZ91HP alloy milling. Appl Comput Sci. 2016;12(4):49-58.

26. Wang J, Zou B, Liu M, et al. Milling force prediction model based on transfer learning and neural network. J Intell Manuf. 2020. https://doi.org/10.1007/s10845-020-01595-w.

27. Dai Y, Zheng X, Chen X, et al. A prediction model of milling force for aviation 7050 aluminum alloy based on improved RBF neural network. Int J Adv Manuf Technol. 2020;110:2493-501. https://doi.org/10.1007/s00170-020-06044-9.

28. Wiciak-Pikuła M, Felusiak A, Chwalczuk T, Twardowski P. Surface roughness and forces prediction of milling Inconel 718 with neural network. In: 2020 IEEE 7th International Workshop on Metrology for AeroSpace. Pisa: MetroAeroSpace; 2020. p. 260-4.

29. Kulisz M, Zagórski I, Korpysa J. The effect of abrasive waterjet machining parameters on the condition of Al-Si alloy. Materials. 2020;13(14):3122. https://doi.org/10.3390/ma13143122.
30. Wu SD, Wu CW, Kin SG, Wang KY, Lee KY. The series analysis using composite multiscale entropy. Entropy. 2013;15:1069-84.

31. Weremczuk A, Borowiec M, Rudzik M, Rusinek R. Stable and unstable milling process for nickel superalloy as abserved by recurrence plots and multiscale entropy. Eksploatacja i Niezawodność. 2018;20(2):318-26. https://doi.org/10.17531/ein. 2018.2.19.

32. ISO 16220:2017-Magnesium and magnesium alloys-Magnesium alloy ingots and castings.

Publisher's Note Springer Nature remains neutral with regard to jurisdictional claims in published maps and institutional affiliations. 